\title{
APPLYING DIFFERENT WIDE-AREA RESPONSE-BASED CONTROLS TO DIFFERENT CONTINGENCIES IN POWER SYSTEMS
}

\author{
A Thesis \\ Submitted to the Faculty \\ of \\ Purdue University \\ by \\ Shahrzad Iranmanesh \\ In Partial Fulfillment of the
Requirements for the Degree \\ of
}

Master of Science in Electrical and Computer Engineering

August 2019

Purdue University

Indianapolis, Indiana 


\section{THE PURDUE UNIVERSITY GRADUATE SCHOOL STATEMENT OF THESIS APPROVAL}

Dr. Steven Rovnyak, Chair

Department of Electrical and Computer Engineering

Dr. Brian King

Department of Electrical and Computer Engineering

Dr. Euzeli Cipriano dos Santos

Department of Electrical and Computer Engineering

Approved by:

Dr. Brian King

Head of Graduate Program 
To my husband Mehdi, my parents Monir, and Mohammad Hassan. 


\section{ACKNOWLEDGMENTS}

First and for the most, I would like to express my sincere gratitude to my advisor Dr. Steven M. Rovnyak for the continuous support of my Master study and my thesis, for his patience, motivation, and immense knowledge. His guidance helped me in all the time of research and writing of this thesis. He shared his knowledge and expertise with me as well as his time and attention to every detail. He was always available to answer my questions.

I would also like to thank my advisory committee members Dr. Brian King, and Dr. Euzeli Cipriano dos Santos for their time and support during the completion of this thesis.

I would like to specially express my appreciation to Dr. Brian King, who granted me with brilliant advice and guidance during this degree whenever I needed help.

I would like to extend my special thanks to the Purdue School of Engineering and Technology, IUPUI, all the faculty and staff who facilitated my thesis work specially to Sherrie Tucker for her kindness in formatting this thesis and for keeping me in mind for every important date or opportunity in the ECE Department.

Finally, I must express my gratitude to my husband Mehdi and to my parents for providing me with unfailing support and continuous encouragement throughout my years of study and through the process of researching and writing this thesis. This accomplishment. 


\section{TABLE OF CONTENTS}

LIST OF TABLES . . . . . . . . . . . . . . . . . . . . vii

LIST OF FIGURES . . . . . . . . . . . . . . . . . . . viii

ABBREVIATIONS ...................... . . . . . . . .

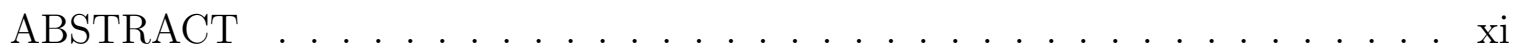

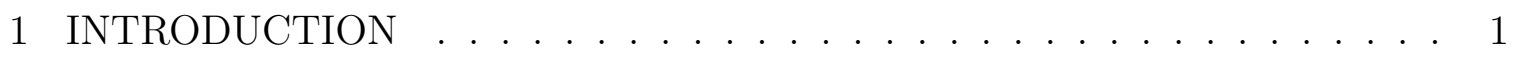

1.1 Problem Statement . . . . . . . . . . . . . . . . . . . . . . 1

1.2 Previous Work . . . . . . . . . . . . . . . 2

1.3 Tools of this thesis . . . . . . . . . . . . . . . 6

1.4 About this thesis . . . . . . . . . . . . . . . 8

2 FEATURE EXTRACTION AND INDICES . . . . . . . . . . . . . 9

2.1 Bus frequency and bus magnitude . . . . . . . . . . . . . . 9

2.2 Integral Square Generator angle (ISGA) . . . . . . . . . . . . . 10

2.3 Calculation of ISBA f . . . . . . . . . . . . . . . . . 11

3 SENSITIVITY ANALYSIS BASED ON ISBA . . . . . . . . . . . . . 13

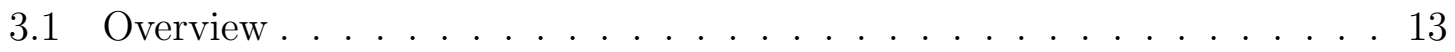

3.2 Result of ISBA Correlations For Two Sample buses . . . . . . . . . . . 13

3.3 Sensitivity analysis of a set of buses . . . . . . . . . . . . . . 14

4 OPTIMIZATION METHOD FOR ONE-SHOT CONTROL . . . . . . . . 21

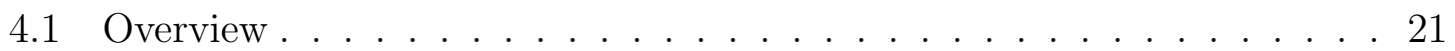

4.2 Gradient Descent Method . . . . . . . . . . . . . . 21

4.2.1 Gradient Descent Result Without the Presence of an Event . . . 23

4.2.2 Gradient Descent Result With the Presence of Event . . . . . . 26

4.3 Particle Swarm Optimization Method . . . . . . . . . . . . . 27

4.3.1 The Result of PSO Algorithm For a Test Event . . . . . . . . . 28 
4.3.2 The Result of the PSO algorithm for a Set of Events . . . . . . 30

5 DECISION TREES FOR CONTROL SELECTION . . . . . . . . . . . 33

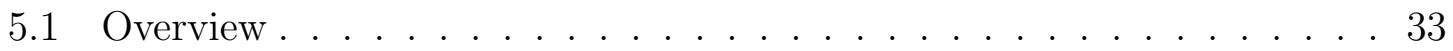

5.2 Control combinations . . . . . . . . . . . . . . . 33

5.3 Data sets . . . . . . . . . . . . . . . . . 34

5.4 Algorithm . . . . . . . . . . . . . . . . . 37

5.5 Implementation of the method . . . . . . . . . . . . . . . 39

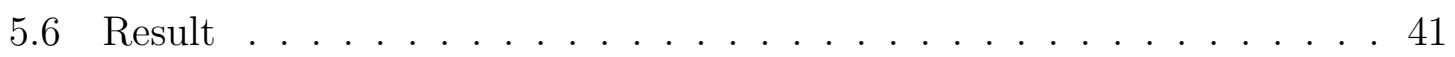

6 CONCLUSION . . . . . . . . . . . . . . . . . . . . 48

REFERENCES . . . . . . . . . . . . . . . . . . . . . 49

A Decision Trees visual representation . . . . . . . . . . . . . . . . 51 


\section{LIST OF TABLES}

Table Page

3.1 The variations of RMSBA on ADELANTO and INTERMT buses . . . . . 14

3.2 Selection of buses with maximum variation in RMSBA . . . . . . . . . 16

3.3 The variations of RMSBA and NStab for the five selected buses . . . . . . 17

3.4 Comparison of three control combinations for the set of events including 480 1-phase events . . . . . . . . . . . . . . . . . 19

3.5 Comparison of three selected control combinations for the set of events including 480 -phase events . . . . . . . . . . . . . . . . . 20

4.1 Ten selected buses for the optimization algorithms . . . . . . . . . . . . . 23

4.2 Gradient Descent result without event . . . . . . . . . . . . . . . 24

4.3 Gradient Descant result with presence of an event . . . . . . . . . . 26

4.4 The result of PSO algorithm for 7 different cases . . . . . . . . . . . . . . 29

4.5 27 buses where control is applied using the PSO algorithm . . . . . . . . . 30

4.6 PSO results for 8 sample events . . . . . . . . . . . . . . . . . . . . 31

5.1 The detail of three control combinations . . . . . . . . . . . . . 35

5.2 The Accuracy results for learning and testing the DTs using a Python code and MATLAB libraries. . . . . . . . . . . . . . . . . . . . 44

5.3 Performance of 3 DTs for control selection 1 phase and 3 phase faults. . . . 44

5.4 Performance of 1 DT for control selection 1 phase and 3 phase faults [13]. . 44 


\section{LIST OF FIGURES}

Figure $\quad$ Page

1.1 Transmission lines for the 176-bus model of WECC [16] . . . . . . . . . 7

2.1 Normalized indices for a specific event happened at 0.55 . . . . . . . . . 12

2.2 Bus voltage magnitudes for a specific event happened at 0.55 . . . . . . . 12

3.1 Number of stabilized control versus delta RMSBA . . . . . . . . . . . . . 15

3.2 Number of stabilized events versus delta RMSBA . . . . . . . . . . . 18

4.1 Minimum J/ISGA versus iteration . . . . . . . . . . . . . . . . 24

4.2 Active power versus iteration . . . . . . . . . . . . . . . 25

4.3 Generator rotor angles before applying control rules of combination 7 . . . 25

4.4 Generator rotor angles after applying control rules of combination 7 . . . 26

4.5 Minimum $\mathrm{J}[\mathrm{k}]$ versus $\mathrm{k}$ for row 1 of Table $4.3 \ldots \ldots$. . . . . . . . . . . 27

4.6 Generator rotor angles during a 3 phase event before applying any control. 32

4.7 Generator rotor angles during a 3 phase event after applying the control found by the PSO algorithm. . . . . . . . . . . . . . . . . . . . 32

5.1 Training data. . . . . . . . . . . . . . . . 36

5.2 Testing data. . . . . . . . . . . . . . . . . . . 38

5.3 A sample decision tree. . . . . . . . . . . . . . . . . . . 40

5.4 The decision tree learning algorithm. a) this function shows the main procedure for building the tree b) this function is finding the best value and column for a data set . . . . . . . . . . . . . . . . . . . 42

5.5 Generator rotor angles during a 3 phase event before applying the control selection algorithm. . . . . . . . . . . . . . . . . . 4 45

5.6 Generator rotor angles during a 3 phase event after applying the control selection algorithm and selecting control combination 2. . . . . . . . 46

5.7 Generator rotor angles during a 3 phase event before applying the control selection algorithm. . . . . . . . . . . . . . . . 46 
Figure

Page

5.8 Generator rotor angles during a 3 phase event after applying the control selection algorithm and selecting control combination 3. . . . . . . . . 47

A.1 DT representation of control combination $1 \ldots \ldots . . . . . . .51$

A.2 DT representation of control combination $2 \ldots \ldots$. . . . . . . 52

A.3 DT representation of control combination $3 \ldots \ldots$. . . . . . . . 53 


\section{ABBREVIATIONS}

WECC Western Electricity Coordinate Council

DT Decision Trees

HIF High Impedance Fault

PMU Phasor Measurement Unit

DG Dispersed Generation

WAMS Wide-Area Monitoring Systems

TSAT Transient Security Assessment Tool

PSAT PowerFlow and Short circuit Assessment Tool

SLD Single Line diagram

UFLS Under Frequency Load Shedding

PSO Particle Swarm Optimization

AI Artificial Intelligent

ISGA Integral Square Generator angle

ISBA Integral Square Bus Angles

RMS Root Mean Square

SLG Single Line to Ground

NN Neural Network 


\begin{abstract}
Iranmanesh, Shahrzad. M.S.E.C.E., Purdue University, August 2019. Applying Different Wide-Area Response-Based Controls to Different Contingencies in Power Systems. Major Professor: Steven Michael Rovnyak.
\end{abstract}

The electrical disturbances in the power system have threaten the stability of the system. In the first step, it is necessary to detect these electrical disturbances or events. In the next step, a proper control should apply to the system to decrease the consequences of the disturbances.

One-shot control is one of the effective methods for stabilizing the events. In this method, a proper amount of loads are increased or decreased to the electrical system. Determining the amounts of loads, and the location for shedding is crucial. Moreover, some control combinations are more effective for some events and less effective for some others. Therefore, this project is completed in two different sections. First, finding the effective control combinations, second, finding an algorithm for applying different control combinations to different contingencies in real time.

To find the effective control combinations, sensitivity analysis is employed to locate the most effective loads in the system. Then in order to find the control combination commands, gradient descent and PSO algorithm are used in this project. In the next step, a pattern recognition method is used to apply the appropriate control combination for every event. The decision tree is selected as the pattern recognition method.

The three most effective control combinations found by sensitivity analysis and PSO method are used in the remainder of this study. A decision tree is trained for each of the three control combinations, and their outputs are combined into an algorithm 
for selecting the best control in real time. Finally, the algorithm is evaluated using a test set of contingencies. The final results reveal a $30 \%$ improvement in comparison to the previous studies. 


\section{INTRODUCTION}

\subsection{Problem Statement}

Providing reliable and stable electrical power is one of the crucial subjects in the operation of the electrical systems. Because of the electrical faults in power stations, damages to electric transmission lines or loss of transmission equipment, the power supply faces many difficulties. On occasion the intensity of some disturbances are high enough to cause the generators losing their synchronization, so a black-out may happen. In some situations, cascading outages may happen in the electrical grid. The Northeast Blackout of 2003 is an example; when a failure to trim trees in Ohio set off a chain of events across the grid that ultimately cut power to 55 million people in eight U.S. states and Canada.

One of the important issues in the electrical system is how to devise techniques for fault detection, and stabilizing them using proper control method. Therefore, after fault detection, selecting an effective combination of control actions and applying it to the system is very important to avoid the spread of faults through the electrical network.

To resolve this issue, the main aim of previous projects and researches was to control and stabilize electrical disturbances in the electrical transmission system [1-3]. In order to achieve this goal, several steps should be accomplished. The first step is event detection, then instability prediction and finally, applying appropriate control to stabilize the events. Pattern recognition methods have been proposed to process wide-area phasor measurements and decide when to apply a combination of one shot controls. However, finding a proper algorithm that categorizes events based on their characteristics and chooses among different control options are not investigated in the recent studies, and it worthwhile to put some efforts into answering this question. 
The main focus of this project is developing a new method to stabilize more events in an electrical transmission model compare to previous researches. Therefore, there are two main questions that should be answered in this project. First, what are the best control options? Second, among all the control combinations, which one is more effective for a specific event? In fact, we want to find an algorithm that can select between different control combinations for different contingencies. Accordingly, we need to use the classification method to classify events based on their characteristics and apply the appropriate control combinations.

\subsection{Previous Work}

Numerous studies have been done with the purpose of detecting disturbances and applying a variety of control methods to stabilize them. Basically, many authors used pattern recognition methods to address the concerns related to electrical disturbances in the power systems.

Pattern recognition methods are proposed for many applications in power system [4-8].The main contribution of [4] is utilizing High Impedance Fault (HIF) detection method based on DTs as pattern recognition method. In [4], only current signals are processed, and six features are extracted as inputs to the DT for detecting HIF. Consequently, the DT algorithm can recognize HIF from normal operation in the power system. In [5], an Empirical Mode Decomposition (EMD) was performed to extract Intrinsic Mode Functions (IMF). The Hilbert Transform is applied as a very effective method for analyzing non-stationary signals. A different pattern recognition approach has been addressed in [6], in which, the authors proposed an island detection and optimal load-shedding scheme for radial distribution systems combined with Dispersed Generation (DG). Using a Probabilistic Neural Network-based (PNN) classifier and exploiting phase-space technique, a passive islanding detection is intro-

duced in [6]. Precisely, they used the Extreme Learning Machine (ELM), which is a neural network with only one hidden layer that randomly assigns values to weights 
and biases and calculates the output analytically. The advantage of ELM is fast training speed. However, since the ELM assigns the initial weight and bias values randomly, there is a problem of consistency in the results. To prevent the inconsistencies, ensemble models were used, so the results from a number of ELMs were used to derive the final result. In [7] two neural network methods have been investigated. Multi-Layer Perceptron (MLP) and Radial Basis Function (RBF) were used for the classification, and MLP was chosen since RBF needs more than 150 neurons in the hidden layer for achieving mean square error close to zero. In [7], a total of thirteen features were extracted such as skewness, kurtosis, form factor, and crest factor to train an artificial neural network for islanding detection. These features are used to detect islanding when there is a deviation in Rate of Change of Frequency (ROCOF). Lidula in [8] employed DT, and Discrete Wavelet Transform (DWT) of the voltage and current to configure the features.

Pattern recognition method has been proposed in $[3,9]$ for stability prediction. Rovnyak and et. al in [3] used DT as a pattern recognition method. The DT predictors in [3] are $\mathrm{R}$ and Rdot, which are apparent resistance and its rate of change measured near the electrical center of Pacific AC Intertie. They created a DT that could be used for response based control but control was not tested in the paper. In [9], the real-time classification was done with Recurrent Neural Networks (RNN), the longterm dependencies were resolved by Long Short Term Memory (LSTM). The pattern recognition method in $[3,9]$, however, does not include any control action.

In some studies, pattern recognition methods are applied to predict islanding in the power system [10]. Diao [10] used the DTs and synchronized phasor measurement to detect loss of synchronism and separate the network into pre-defined islands. A different approach is used for training of the DT in [10]; in fact, one DT is trained for each contingency instead of training one DT for all of the contingencies. Diao [10] used the voltage phase angles measurement of high voltage buses, and for each phase angle variables, they defined six features. 
Some of the studies proposed islanding control method after instability prediction to maintain the frequency $[11,12]$. The island management method proposed in [12] can maintain synchronism within each island. The feasible islanding interval is studied in [11] for applying island control method. The island control method can be considered as a backup for the control method in the current study.

In some other studies, pattern recognition is used to order control that keeps synchronization and avoids the need for islanding. Gao and et al. [2] used two different approaches for DT construction process, and one of the methods resulted in a smaller region of feature space that is stable. The smaller region of space that is stable results in earlier detection of instability. Gao and et al. [2] used 68 features as predictor calculated or measured using the Phasor Measurement Unit (PMU). One of the main contributions of [2] is that they used the one-shot control to avoid the loss of synchronism occurred by the events rather than splitting the electrical grid into islands. The algorithm in [2] really does order control that keeps synchronization and avoids need for islanding. Mei and et.al [1] suggested a method to develop response-based decision trees to activate control for stabilizing the events. The control used in [1,2] is a fixed combination of power changes in four buses, but in the current study, the algorithm can select among control options. In [13], the authors used Wide-area Monitoring Systems (WAMS) to communicate the phase angle data measured by Phasor Measurements Units (PMU). They used pattern recognition methods like DT, to apply a one-shot control. They employed the combination of separate event detection and control DTs for transient stability control. Their control actions included disconnection of costly generation and load. Moreover, to train the DT, they applied some old and new indices. Eventually, the results show a higher rate of success stabilizing events using one shot control. The control used in [13] is a fixed combination of power changes in four buses. A novel Under Frequency Load Shedding (UFLS) algorithm is used in [14]. In [14] the authors proposed a three stages scheme as a new centralized 
adaptive load shedding. The first stage includes analyzing the required data and sizing the reactive power. In the second step, the optimal amount of loads and their locations are specified. Finally, the third stage includes determining the event type.

A new control strategy is proposed in [15], which can choose between two sets of control rules. In addition to DTS for event detection and instability prediction, the author used a third decision tree to apply a proper one-shot control, so the number of stabilized cases was improved to 30 events. One of the drawbacks with this project is that they found the control options by trial and error method. In the current project, on the other hand, numerical methods are used to find a variety of control options.

The main goal of the current thesis project is to stabilize faults or electrical disturbances in the electrical transmission system. To begin, previous works of Rovnyak and et.al are studied [1-3,13]. Furthermore, the goal is to increase the number of stabilized events in comparison to previous studies. In order to achieve this goal, we developed techniques for controlling events. Moreover, instead of two control options, three control options are selected in the current study.

The control application area of this project is referred to as one-shot control, which is a proposed control method to prevent the loss of synchronism and preserving the security of both transmission and generation during disturbances. The conventional one-shot control determines the size and the number of the load to change, and regardless of the location and intensity of the disturbance applies a constant load scheme. However, in the current project, a new algorithm is developed, that can apply different control options to different contingencies.

For developing this algorithm, this project is established in three steps. In the first step, a sensitivity analysis has been developed to locate the most effective buses. Besides, an optimization technique is exploited for finding the proper amount of loads to be shed and determining the location of load changes. Particle Swarm Optimization (PSO) and Gradient Descent are used as two optimization methods. MATLAB 
programming is employed for developing the algorithm, and TSAT is used for transient analysis. The results of optimization algorithms help us to find different control schemes.

In the last step, a pattern recognition method has been applied to create decision criteria for deciding to actuate control and select one of several control combinations. Basically, the pattern recognition methods are Artificial Intelligent (AI) methods.

The study model in this project is the Western Electricity Coordinating Council (WECC) as represented in Figure 1.1. Different types of 1-phase and 3-phase disturbances are simulated using TSAT, and the data are analyzed using MATLAB. Various types of features are calculated based on the recorded data. In addition, two sets of Training and and Test data are are produced to train and test the controlling algorithm technique.

\subsection{Tools of this thesis}

Two power system software tools are used in this project. Transient Security Assessment Tool (TSAT) and PowerFlow and Short circuit Assessment Tool (PSAT).

TSAT is a software tool, established by Powertech Labs Inc., for transient analysis of power systems. Using the benefits of Transient Security Assessment (TSA), this software has features for online and off-line TSA analysis. It is a nonlinear time-domain simulation engine, which has the ability to produce precise responses to various types of contingencies happening in large interconnected power systems. It has various applications such as time-domain simulation for processing large and complex power system models and determination of system stability [17].

On the other hand, PSAT is another software tool by Powertech Labs Inc. It is a graphical program for building and adjusting power flow data by drawing Single Line diagram (SLD) and solving power flow. The solution of power flow analysis is 


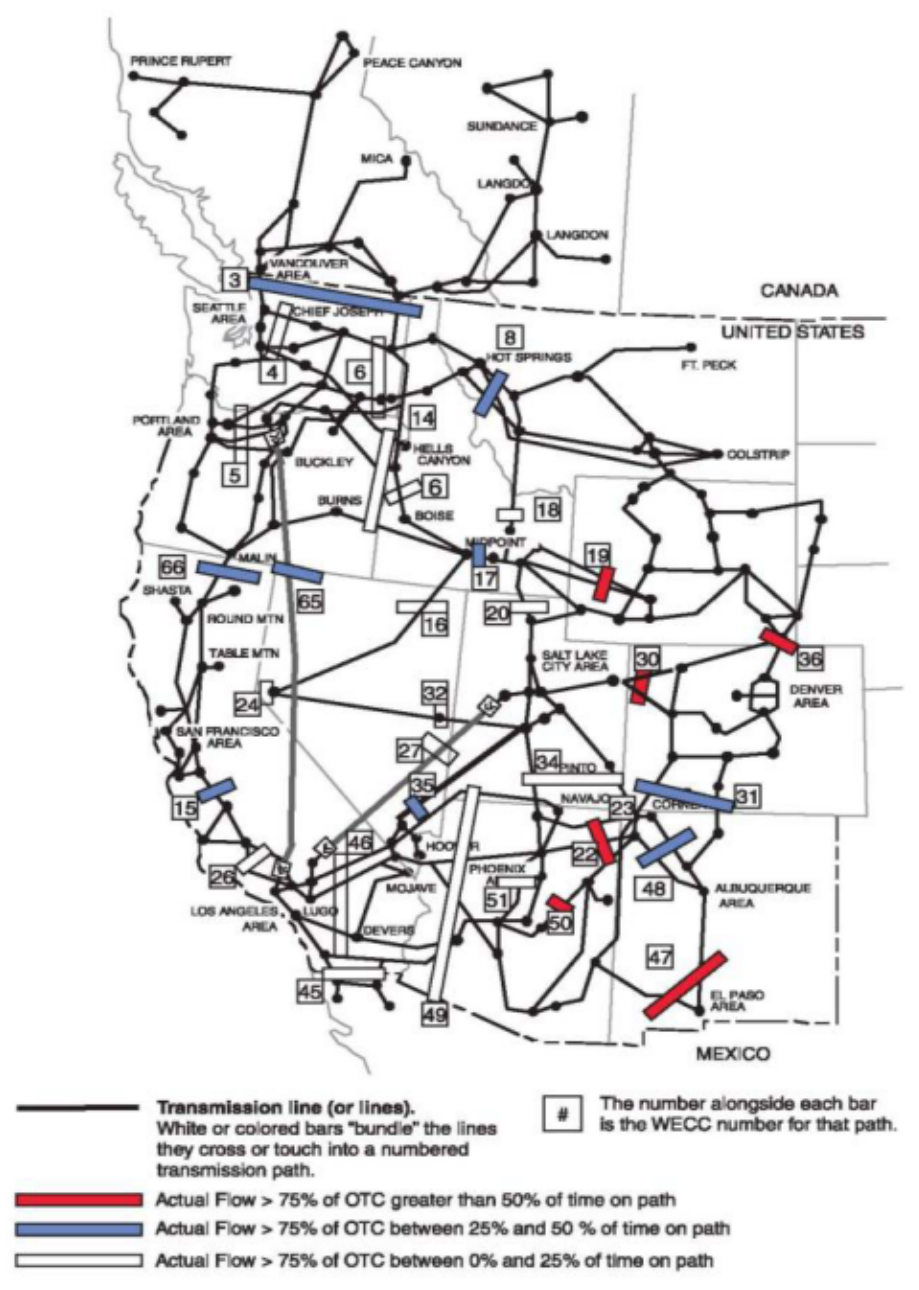

Fig. 1.1.: Transmission lines for the 176-bus model of WECC [16]

represented both in tables and on diagrams. In addition to the modification of power flow models, it has applications in harmonic analysis, short-circuits, and contingency assessment [18]. 


\subsection{About this thesis}

In the next chapter, applied features and related equations are evaluated. In the third chapter, sensitivity analysis is used to locate the most effective buses. In the fourth chapter, the optimization method and the related results are described. In the fifth chapter, the detail of the AI method, which is DT is explained, and the final results related to this project are assessed. 


\section{FEATURE EXTRACTION AND INDICES}

A variety of Indices or predictors can be used for Sensitivity analysis and pattern recognition method in this project. Some of the studies used only two predictors since using a two-dimensional feature space that can be visualized like R-Rdot application in [1] is easier to study. In another study [2], voltage angle, voltage magnitude and their rate of change are exploited as the predictors. In this project, a large number of predictors are calculated from the measurements. A reduced set of predictors is obtained after training several DTs with all the predictors. The subset consists of predictors that appear near the root nodes of DTs.

\subsection{Bus frequency and bus magnitude}

The first set of variables are bus voltage magnitudes and bus voltage angles that can be measured by PMU installed on the buses in the network. For each bus, there are the voltage magnitude and angle variables plus the derivative of each of them. If there are PMUs installed in $\mathrm{N}$ buses, then $4 \mathrm{~N}$ elements can be added to the input vector of the classification method. In this study, $N=17$. The derivative of voltage angles and magnitude can be calculated from the difference of samples. For example, if the sampling of the simulation is $1 / 30 \mathrm{~Hz}$, the derivative of the bus voltage angle, which is the frequency of the voltage is calculated using (2.1)

$$
B F[i]=30(B A[i]-B A[i-1])
$$

In (2.1), BF is bus frequency, BA is bus angle, and $i$ shows the number of sam-

ple. The average and variance of bus magnitude are two other indices that can be calculated using (2.2) and (2.3). 


$$
\begin{gathered}
B M a v g[k]=\sum_{i} \frac{\left|V_{i}[k]\right|}{17} \\
B M v a r[k]=\sum_{i} \frac{\left(\left|V_{i}[k]\right|-B M a v g[k]\right)^{2}}{17}
\end{gathered}
$$

The derivative of BMavg and BMvar can be calculated from point to point differences between samples 30 times per second.

$$
\begin{aligned}
& \text { BMavgdot }[k]=30(B \operatorname{Mavg}[k]-B \operatorname{Mavg}[k-1]) \\
& \text { BMvardot }[k]=30(B \operatorname{Mvar}[k]-B \operatorname{Mvar}[k-1])
\end{aligned}
$$

Aside from the individual generator angles and bus voltage angles measured in the system, the following indices are calculated and implemented in the classification algorithm of this project.

\subsection{Integral Square Generator angle (ISGA)}

One of the effective indices that can be applied for the classification objective is Integral Square Generator angle (ISGA). This index is a coherency based index that can be used to judge the severity of stable and unstable events in the simulations. Multi-machine Integral Square Generator Angle (ISGA) index can be defined as follow

$$
I S G A=\int_{0}^{T} M_{i}\left(\delta_{i}(t)-\delta_{c o a}(t)\right)^{2} d t
$$

where $M_{i}$ are the machine inertias, $\delta_{i}(t)$ are the generator angles as a functions of time and $\delta_{\text {coa }}(t)$ is the center of angle, which is evaluated as follows [19]

$$
\delta_{\text {coa }}(t)=\frac{\sum_{i} M_{i} \delta_{i}(t)}{\sum_{i} M_{i}}
$$




\subsection{Calculation of ISBA}

Unlike the generator angle, bus angles are discontinuous at -180 degrees and 180 degrees. Since bus angles do not go beyond the -180 to 180 degrees range, if a bus angle goes beyond this interval, it wraps around to the opposite side and results in a 360 degrees difference. By adding and subtracting 360 degrees and comparing them to the thresholds values, this problem can be resolved.

In addition, in real time, it is not possible to measure generator angles directly, bus voltage angles from PMUs are used in the calculation of ISGA, so the new index is Integral Square Bus Angles (ISBA). ISBA can express the overall stress on the system [12]. The next step is finding the Square Bus Angle (SBA) Index that can be calculated using equation (2.8).

$$
S B A[k]=\sum_{i} M_{i}\left(\Theta_{i}[k]-\Theta_{c o a}[k]\right)^{2}
$$

where $M_{i}$ is chosen to weight angles from different locations, $\Theta_{i}[k]$ represents the bus angles measured by PMUs and $\Theta_{\text {coa }}$ is calculated as follows

$$
\Theta_{\text {coa }}[k]=\frac{\sum_{i} M_{i} \Theta_{i}[k]}{\sum_{i} M_{i}}
$$

In the current thesis, it is possible to consider equal weights $M_{i}$ to all monitored buses. Another index, which is used in this study is the derivative of the SBA that is

$$
S B A d o t[k]=30(S B A[k]-S B A[k-1])
$$

According to [15], instead of integrating SBA over a sliding window we can use a low pass filter with a transfer function $\mathrm{G}(\mathrm{s})=6 /(\mathrm{S}+6)$. According to (2.8), ISBA has a cumulative nature, so the final value has the impact of all samples in it.

The derivative of the index values for a 3-phase short circuit fault is shown in Figure 2.1. It started at $0.55 \mathrm{~s}$ and cleared at $0.67 \mathrm{~s}$, and its location is a line between HANFORD and JOHN DAY buses. This figure shows the variation of BMvardot, BMavgdot, and SBAdot. As we can see BMvardot and sbadot are increasing during 
the presence of fault and after clearing fault these indexes are reducing. However, bmavgdot has a different behaviour. Figure 2.2 shows the variation of 17 PMU bus voltage magnitudes during the aforementioned short circuit event.

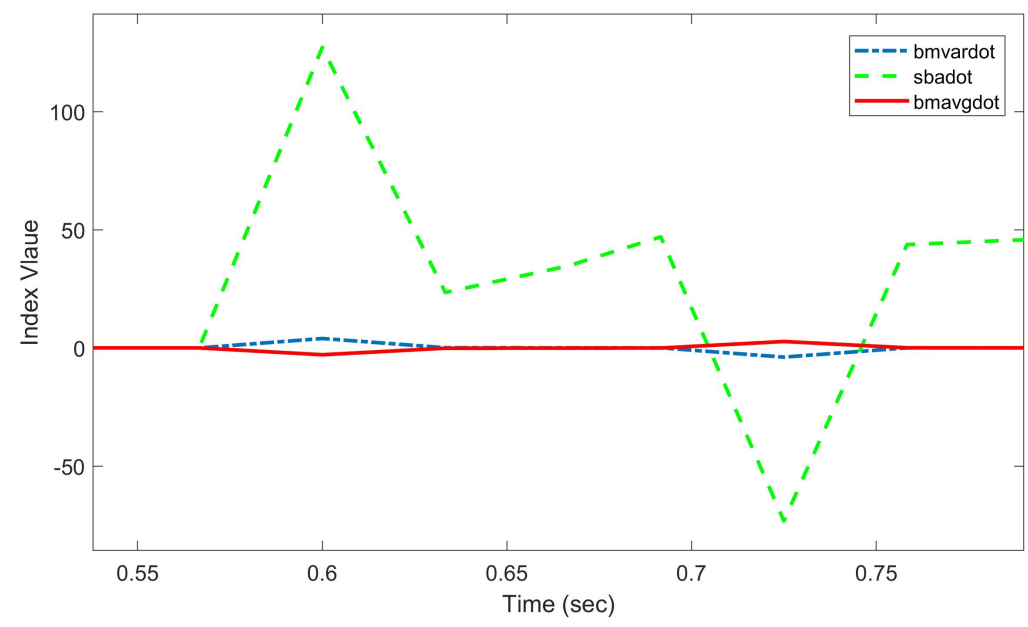

Fig. 2.1.: Normalized indices for a specific event happened at 0.55

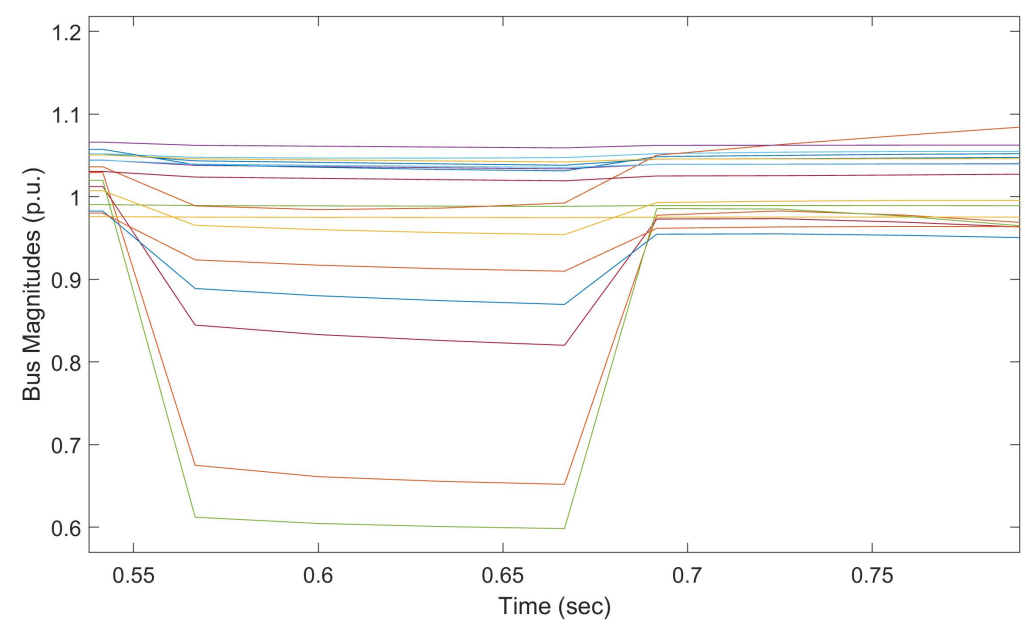

Fig. 2.2.: Bus voltage magnitudes for a specific event happened at 0.55 


\section{SENSITIVITY ANALYSIS BASED ON ISBA}

\subsection{Overview}

In this chapter, the correlation of ISBA and the load variations on different buses are analyzed. In the previous chapter, ISBA was calculated using the equation (2.8). If we take the square root of ISBA, we get the root mean square (RMS) bus angle, which is called RMSBA.

\subsection{Result of ISBA Correlations For Two Sample buses}

In the first part of this chapter, we tried to find the correlation between RMSBA and the number of stabilized events. Therefore, two buses are selected ADELANTO 500 and INTERMT 345. If we add or reduce the loads on these buses for $200 \mathrm{MW}$, four different control combinations can be selected. In each simulation, to see the effect of these control commands on the model, first, we have to run the simulation without fault and calculate RMSBAbase. Then the controller is added; new RMSBA is calculated, and its difference from RMSBAbase is determined.

In the next step, the test set including 480 1-phase faults are exploited, and each of the four control combinations is simulated. We considered the last sample of ISBA as the final value for RMSBA because of its cumulative nature. In addition, we used each of these control combinations as one-shot control in the program that runs all the simulations in test sets. Then the number of simulations stabilized for each control combination, and also the number of simulations destabilized is evaluated for each control combination. 
Table 3.1 shows the result of the simulation. There are four different binary situations; 00 means $200 \mathrm{MW}$ load is reduced from bus 1 and bus 2. 01 means the 200 MW load reduced on bus 1, and 200 MW load is added to bus 2. deltaRMSBA shows the difference of RMSBabase and RMSBA. Nstab is the difference between the number of Stabilized events and number of destabilized events.

Table 3.1.: The variations of RMSBA on ADELANTO and INTERMT buses

\begin{tabular}{|l|l|l|l|l|l|l|}
\hline & RMSBAbase & RMSBA & deltaRMSBA & Stabilized & destabilized & Nstab \\
\hline 00 & 32.1753 & 29.198 & -2.9772 & 6 & 0 & 6 \\
\hline 01 & 32.1753 & 31.0444 & -1.1308 & 0 & 0 & 0 \\
\hline 10 & 32.1753 & 33.6327 & 1.4575 & 1 & 0 & 1 \\
\hline 11 & 32.1753 & 34.0575 & 1.8823 & 0 & 2 & -2 \\
\hline
\end{tabular}

Figure 3.1 illustrates the correlation of deltaRMSBA and Nstab. We can see there is a strong negative correlation between these two indexes. Therefore, the stability of a system will be increased by reducing the RMSBA index.

\subsection{Sensitivity analysis of a set of buses}

The goals of this section are first finding the sensitivity of RMSBA to all of the load changes at all the buses, and second finding a control combination based on the results of sensitivity analyses. The last aim is to obtain 3 combinations and a pattern recognition to decide which action to take that will stabilize more events in the test set than any single control combination.

We added and reduced a 200 MW load to all load buses, which are 147 buses, and deltaRMSBA is calculated in each case because Delta RMSBA can be considered as the sensitivity of each bus to load variations. Table 3.2 shows a selection of load buses with maximum deltaRMSBA. We can see from this Table, some of the buses can reduce RMSBA when we increased load on them like MONTANA 500, and some 


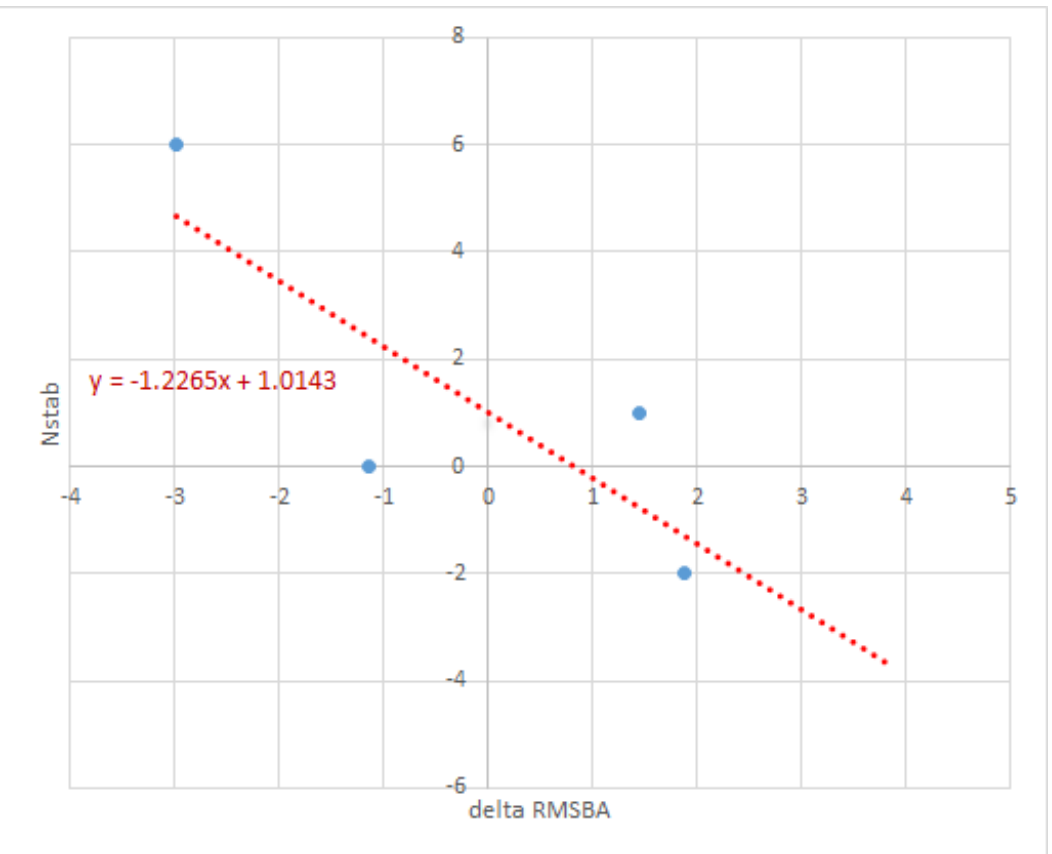

Fig. 3.1.: Number of stabilized control versus delta RMSBA

of them can reduce RMSBA by decreasing the amount of load on them, like MIDWAY 200; the only exception is CANALB 500 that decreases RMSBA for both increasing load and decreasing load situation. The negative number of each row shows the priority of selecting that bus for control objective, so the more negative the higher priority.

To be economical, we selected only five buses for the first control combination in this section as follow.

- MONTANA 500

- MIDWAY 200

- NAVAJO1 500

- MOHAVE 500

- CA230TO 
Table 3.2.: Selection of buses with maximum variation in RMSBA

\begin{tabular}{|c|l|c|c|}
\hline & Bus Name & DeltaRMSBA (-200) & DeltaRMSBA (200) \\
\hline 1 & MONTANA 500 & 129.56 & -5.14 \\
\hline 2 & MIDWAY 200 & -2.42 & 2.00 \\
\hline 3 & NAVAJO1 500 & -2.33 & 2.03 \\
\hline 4 & FOURCOR2 500 & -2.30 & 2.01 \\
\hline 5 & MOHAVE 500 & -2.30 & 2.01 \\
\hline 6 & NAVAJO 500 & -2.2958 & 1.98 \\
\hline 7 & WESTWING 500 & -2.2954 & -1.98 \\
\hline 8 & CANALB 500 & -2.14 & -1.92 \\
\hline 9 & CA230 230 & 125.26 & -1.69 \\
\hline 10 & CA230TO 230 & 64.29 & -1.32 \\
\hline 11 & CANADA 500 & 1.65 & \\
\hline
\end{tabular}

In order to find the correlation between deltaRMSBA and the number of stabilized events, we used the same method defined in the previous section. As we have five buses, and there are 2 states 1 or 0 for each bus, so there are $2^{5}$ different modes. In this example, 0 shows positive load change and 1 shows negative load change. For example, $\mathrm{M}=01110$ represents the increasing load at MONTANA 500 and CA230TO 230; decreasing load at MIDWAY 200, NAVAJO1 500 and MOHAVE 500. Table 3.3, shows delta RMSBA and the number of stabilized events for different control combinations. As we are expecting from the results of Table 3.2, the best control mode is 01110. Since according to Table 3.2, MONTANA, and CA230TO returned a negative value for RMSBA only when the load is added to them. However, for MIDWAY, NAVAJO1, and MOHAVE the opposing situation has happened. 
Table 3.3.: The variations of RMSBA and NStab for the five selected buses

\begin{tabular}{|c|c|c|c|c|c|c|}
\hline Control mode & RMSBA-base & RMSBA & delta RMSBA & Stab & destab & Nsatb \\
\hline 00000 & 32.18 & 31.45 & -0.72 & 1 & 2 & -1 \\
\hline 00001 & 32.18 & 228.21 & 196.04 & 0 & 6 & -6 \\
\hline 00010 & 32.18 & 27.15 & -5.02 & 14 & 0 & 14 \\
\hline 00011 & 32.18 & 191.73 & 159.55 & 0 & 6 & -6 \\
\hline 00100 & 32.18 & 27.44 & -4.74 & 9 & 2 & 7 \\
\hline 00101 & 32.18 & 197.13 & 164.95 & 0 & 6 & -6 \\
\hline 00110 & 32.18 & 23.52 & -8.65 & 22 & 0 & 22 \\
\hline 00111 & 32.18 & 141.29 & 109.12 & 0 & 6 & -6 \\
\hline 01000 & 32.18 & 27.36 & -4.81 & 8 & 1 & 7 \\
\hline 01001 & 32.18 & 200.86 & 168.69 & 0 & 6 & -6 \\
\hline 01010 & 32.18 & 23.44 & -8.74 & 23 & 0 & 23 \\
\hline 01011 & 32.18 & 134.21 & 102.04 & 0 & 6 & -6 \\
\hline 01100 & 32.18 & 23.86 & -8.32 & 20 & 1 & 19 \\
\hline 01101 & 32.18 & 142.45 & 110.27 & 0 & 6 & -6 \\
\hline 01110 & 32.18 & 20.75 & -11.43 & 26 & 0 & 26 \\
\hline 01111 & 32.18 & 91.84 & 59.66 & 0 & 6 & -6 \\
\hline 10000 & 32.18 & 34.48 & 2.30 & 0 & 6 & -6 \\
\hline 10001 & 32.18 & 438.96 & 406.78 & 0 & 6 & -6 \\
\hline 10010 & 32.18 & 29.27 & -2.91 & 0 & 6 & -6 \\
\hline 10011 & 32.18 & 149.81 & 117.64 & 0 & 6 & -6 \\
\hline 10100 & 32.18 & 29.75 & -2.42 & 0 & 6 & -6 \\
\hline 10101 & 32.18 & 140.74 & 108.56 & 0 & 6 & -6 \\
\hline 10110 & 32.18 & 24.87 & -7.31 & 0 & 6 & -6 \\
\hline 10111 & 32.18 & 113.04 & 80.86 & 0 & 6 & -6 \\
\hline 11000 & 32.18 & 29.56 & -2.62 & 0 & 6 & -6 \\
\hline
\end{tabular}


Continued Table 3.3

\begin{tabular}{|l|l|l|l|l|l|l|}
\hline 11001 & 32.18 & 150.81 & 118.63 & 0 & 6 & -6 \\
\hline 11010 & 32.18 & 24.69 & -7.49 & 0 & 6 & -6 \\
\hline 11011 & 32.18 & 114.06 & 81.88 & 0 & 6 & -6 \\
\hline 11100 & 32.18 & 25.27 & -6.90 & 0 & 6 & -6 \\
\hline 11101 & 32.18 & 125.96 & 93.78 & 0 & 6 & -6 \\
\hline 11110 & 32.18 & 22.00 & -10.18 & 0 & 6 & -6 \\
\hline 11111 & 32.18 & 87.58 & 55.41 & 0 & 6 & -6 \\
\hline
\end{tabular}

Figure 3.2, illustrates the correlation of delta RMSBA and Nstab for positive value of Nstab in Table 3.3. As we concluded in the previous section, there is a strong negative correlation between these two indexes. Therefore, the stability of a system increases by reducing the RMSBA index.

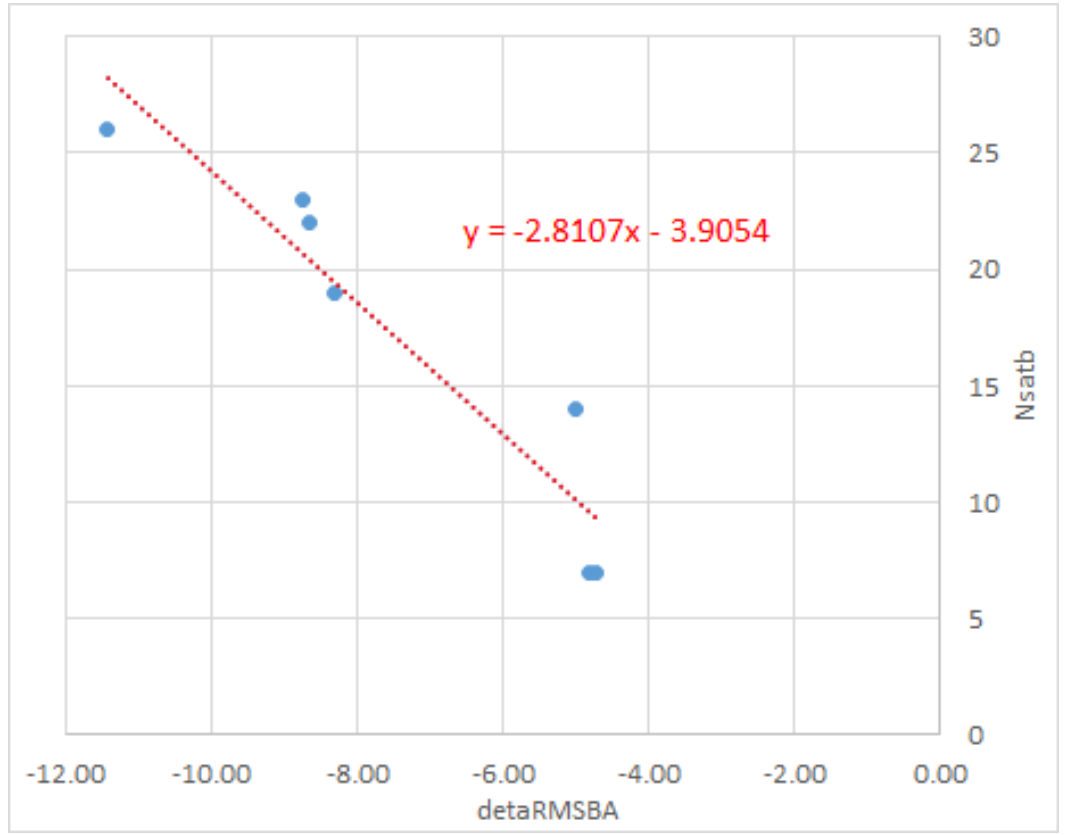

Fig. 3.2.: Number of stabilized events versus delta RMSBA 
For the next step, two different combinations used in the previous researches were selected. Then their performances are compared with control combination 01110 found in this section. We refer to 01110 as control combination 1. Control combination 2 was used in $[2,20]$. It is a one-shot control action consist of a step changes in the real power injections at pairs of AC buses; one $500 \mathrm{MW}$ fast load increase is applied to COLSTRIP in MONTANA and a bus in CANADA. Besides, step load decreases occur at two buses around the southern ends of the two HVDC lines. Control combination 3 is used in [15], and basically, it is a step load increases at CANADA and MONTANA, and step load decreases at RINALDI and SYLMARLA. Besides, a generator is disconnecting at CANADA.

Table 3.4 shows the simulation results of three control combinations for 480 1phase disturbances. In all of the simulation, the same decision tree used in [13] is implemented. The configuration for control with event detection uses BMvardot $<-0.5$ for event detection and Busfrequency $9>50$ for control actuation. As we can see in this table, control combination 1 introduced in this section returned better results in comparison with two other combinations. Table 3.5 illustrates the performance of the control combinations for a set of 480 3-phase events.

Table 3.4.: Comparison of three control combinations for the set of events including 480 1-phase events

\begin{tabular}{|l|l|l|l|}
\hline $480-1$ phase events & Control combination 1 & Control combination 2 & Control combination 3 \\
\hline Events Stabilized & 26 & 21 & 21 \\
\hline Events Destabilized & 0 & 0 & 0 \\
\hline Events Keep stable & 276 & 276 & 276 \\
\hline Events Keep unstable & 178 & 183 & 183 \\
\hline Events Controlled & 96 & 96 & 96 \\
\hline Control Unnecessary & 6 & 6 & 6 \\
\hline Events Not Detected & 12 & 12 & 12 \\
\hline Mean Control Time & 0.77 & 0.77 & 0.77 \\
\hline Success Rate & 0.271 & 0.219 & 0.219 \\
\hline
\end{tabular}


Table 3.5.: Comparison of three selected control combinations for the set of events including 480 3-phase events

\begin{tabular}{|l|l|l|l|}
\hline $480-3$ phase events & Control combination 1 & Control combination 2 & Control combination 3 \\
\hline Events Stabilized & 32 & 29 & 29 \\
\hline Events Destabilized & 0 & 0 & 0 \\
\hline Events Keep stable & 250 & 250 & 250 \\
\hline Events Keep unstable & 198 & 201 & 201 \\
\hline Events Controlled & 113 & 113 & 113 \\
\hline Control Unnecessary & 13 & 13 & 13 \\
\hline Events Not Detected & 54 & 54 & 54 \\
\hline Mean Control Time & 0.912 & 0.912 & 0.912 \\
\hline Success Rate & 0.283 & 0.257 & 0.257 \\
\hline
\end{tabular}

In the next section, we want to determine whether there are events stabilized by one control that is not stabilized by any other control. To answer this question, we should save stabilized events for each control combination in a separate file. For example, this file for control combination 2 in Table 3 includes 21 events. If we run the file for control combination 1 , and the number of stabilized events was 21 , control combination 1 can control all stabilized events of control combination 2 . While if the number of stabilized events become less than 21, it shows some of the events can be stabilized by control combination 2 , and control combination 1 cannot stabilize them.

In [15] two different controls are exploited using a third decision tree to choose between two controls, so the number of stabilized cases was improved. The maximum number of stabilized events using both controls in [15] is 30 .

In the current research, after saving 3 files including stabilized events, the simulation results showed all the events, stabilized using control combination 2 and control combination 3 can be stabilized by control combination 1. According to Table 3.5, all the stabilized events are 32, which is higher than the previous researches. Success rate also improved in this section. 


\section{OPTIMIZATION METHOD FOR ONE-SHOT CONTROL}

\subsection{Overview}

In this chapter, two different methods are exploited to determine adequate loads for the control rules. In fact, the main goal is to identify the amount of load to be shed and the buses that applying these changes. The first method is a proposed gradient descent and the second method is Particle Swarm Optimization (PSO). The gradient method is explained in two sections: Section 1: Gradient descent without the presence of an event; Section 2: Gradient descent with the presence of an event. The rest of the chapter is explaining the detail of each method and the results related to them.

\subsection{Gradient Descent Method}

We proposed a numerical gradient descent equation for evaluating the amount of active power on each bus included in the control combination rules. If the initial matrix of active power for $\mathrm{N}$ buses is considered as (4.1) for the first iteration, ISGA for any rows of $X_{0}$ is evaluated as matrix $J_{i}$ in (4.2). The index $i$ shows the iteration number.

$$
X_{0}=\left[\begin{array}{ccccc}
0 & 0 & 0 & \cdots & 0 \\
50 & 0 & 0 & \cdots & 0 \\
0 & 50 & 0 & \cdots & 0 \\
\vdots & \vdots & \ddots & \vdots & \vdots \\
0 & 0 & 0 & \cdots & 50
\end{array}\right]_{(N+1) \times N}
$$




$$
J_{i}=\left[\begin{array}{c}
J_{i 0} \\
J_{i 1} \\
J_{i N}
\end{array}\right]_{(N+1) \times 1}
$$

In every iteration, each row of matrix $\mathrm{X}$ is updated separately, based on the matrix $\mathrm{J}$, which is used in the following equation (4.3). The first index of each element shows the iteration number, and the second number of the index shows the bus number. The function sign shows the direction of each variable for the next step.

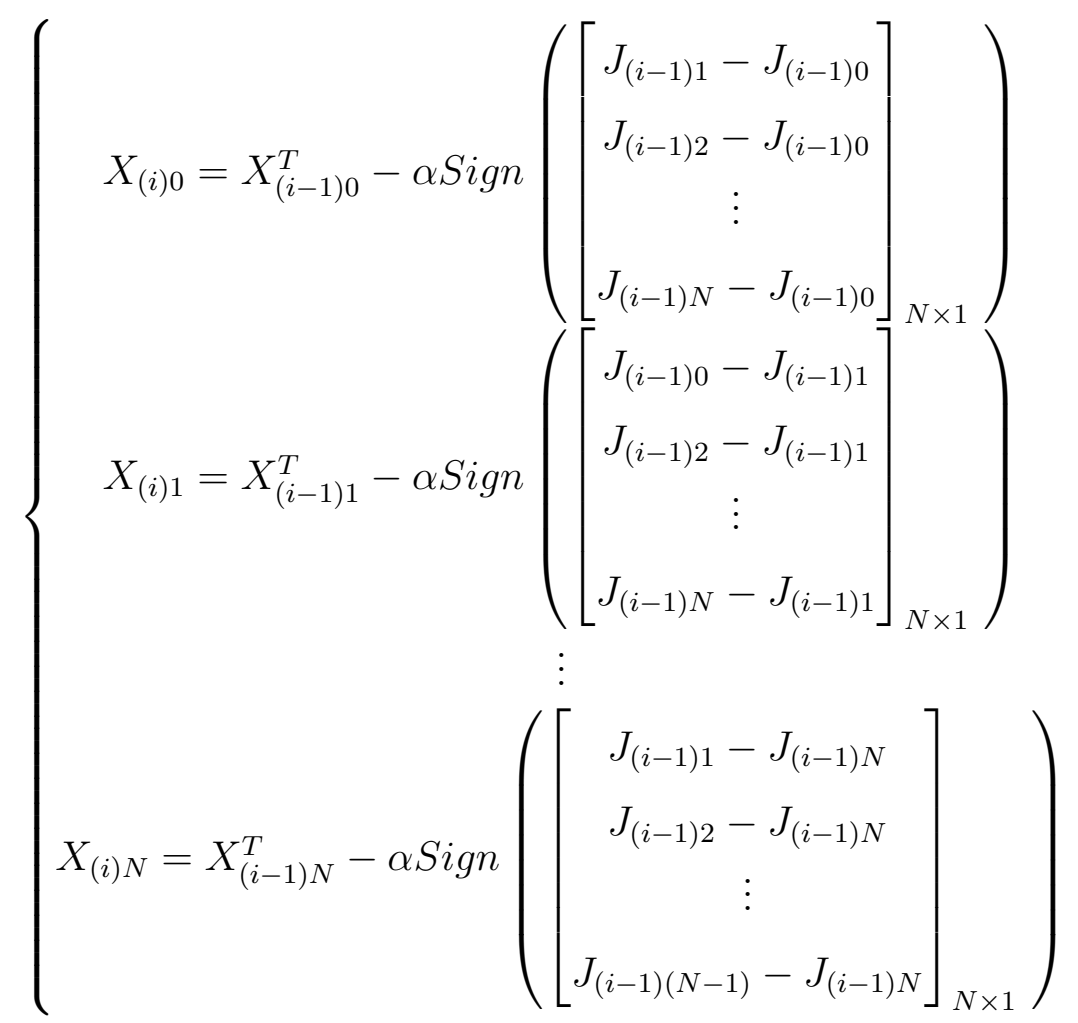

$X_{(i) 0}, X_{(i) 1}, \ldots, X_{(i) N}$ are the vectors showing the rows of matrix $\mathrm{X}$, and the subtitle $i$ shows the iteration number of the vectors. $\alpha$ is the step size of the gradient method. The sign matrix is defined as (4.4).

$$
\operatorname{sign}(x)=\left\{\begin{array}{cc}
-1 & x>0 \\
0 & x=0 \\
1 & x<0
\end{array}\right.
$$


In the next section, the gradient method is considered in two different cases. First, without applying an event, second in the presence of an event. For both cases, ten buses have been chosen based on the sensitivity analysis to the ISGA in Chapter 3, and they are shown in Table 4.1.

Table 4.1.: Ten selected buses for the optimization algorithms

\begin{tabular}{|c|c|c|c|c|}
\hline MONTANA & MIDWAY & NAVAJO1 & MOHAVE & CA230 \\
\hline VALLEY & RINALDI & FOURCORE2 & WESTWING & CANALB \\
\hline
\end{tabular}

\subsubsection{Gradient Descent Result Without the Presence of an Event}

In the first step, we used the Gradient descent algorithm when there was no event in the system. Table 4.2 illustrates the results related to this section. Each row shows the result for one simulation of the algorithm that can be considered as a different combination. Eleven various combinations are represented in this table. The goal is to find the best values for the Step size, the number of iteration, the simulation time and the value of the initial matrix.

In Table 4.2, Jmin is the most recent minimum ISGA evaluated in every run of the algorithm. alpha shows the step size in the gradient algorithm. It - dec two numbers; the first number is the iteration number in which the step size is decreasing, and the second number is the amount of reduction. Iter is the maximum number of iteration for each simulation. Initial is the value of the initial matrix explained in the previous section. ActivePowerforbus $1-10$ shows the value of active power for bus 1 to bus 10 when the ISGA is minimum, so it shows the best solution for each combination.

Figure 4.1 illustrates the variation of minimum $\mathrm{J}$ versus the iteration number for combination 7. As we can see, it starts at 3.945, and it reduces to 3.915 after 60 iterations. 
Table 4.2.: Gradient Descent result without event

\begin{tabular}{|c|c|c|c|c|c|c|c|}
\hline & J min & alpha & It - dec & It & Sim Time & Initial & Active Power for bus 1-10 \\
\hline 1 & 3.89 & 10 & $0-0$ & 100 & $12 \mathrm{~s}$ & 50 & $110-930-15050-130-690-810-8305050$ \\
\hline 2 & 2.35 & 10 & $0-0$ & 100 & $20 \mathrm{~s}$ & 50 & $-260-440-42060600-620-440-440-420$ \\
\hline 3 & 3.89 & 10 & $0-0$ & 150 & $12 \mathrm{~s}$ & 20 & $170-10-1230270310190-1210-1210290330$ \\
\hline 4 & 3.91 & 10 & $70-10$ & 150 & $12 \mathrm{~s}$ & 20 & $140.1-587.1-216.5-141.8-161.8-81.8-449.1-487.1132 .9112 .9$ \\
\hline 5 & 3.89 & 20 & $70-10$ & 100 & $12 \mathrm{~s}$ & 20 & $120200-130016020080-1240-1240480320$ \\
\hline 6 & 7.78 & 20 & $70-10$ & 130 & $6 \mathrm{~s}$ & 40 & $-260-980-460220220180-900-900140100$ \\
\hline 7 & 3.91 & 10 & $50-10$ & 100 & $12 \mathrm{~s}$ & 50 & $-40.743 .6-589.510 .521 .23 .1-529.5-589.5-589.522 .4$ \\
\hline 8 & 3.89 & 10 & $70-5$ & 120 & $12 \mathrm{~s}$ & 50 & $69.3-831.3-433.5157 .8131 .35-631.3-671.3-791.3-19.1-199.1$ \\
\hline 9 & 7.78 & 10 & $70-5$ & 140 & $6 \mathrm{~s}$ & 50 & $-276.2-783.6-595.2244 .1243261 .1-723.6-743.6-343.6-255.2$ \\
\hline 10 & 7.79 & 10 & $60-5$ & 200 & $6 \mathrm{~s}$ & 50 & $-249.04-685.7-560.8274 .6279 .5291 .9625 .7-645.7-245.7-220.8$ \\
\hline 11 & 3.91 & 10 & $60-10$ & 160 & $12 \mathrm{~s}$ & 50 & $-110-50-690-70-17.9-18.4-630-690-69035.8$ \\
\hline
\end{tabular}

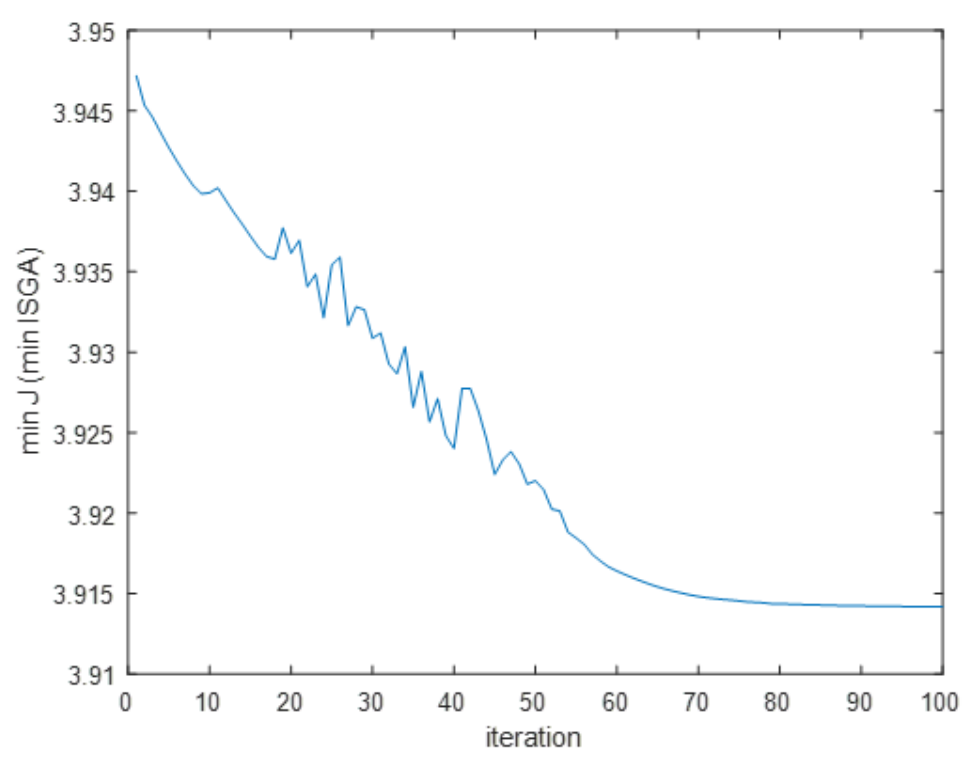

Fig. 4.1.: Minimum J/ISGA versus iteration

Figure 4.2 displays how active power related to each bus is changing in every iteration. As we can see, the active power for any of the buses reached an almost fixed value as the number of iteration is increasing. 


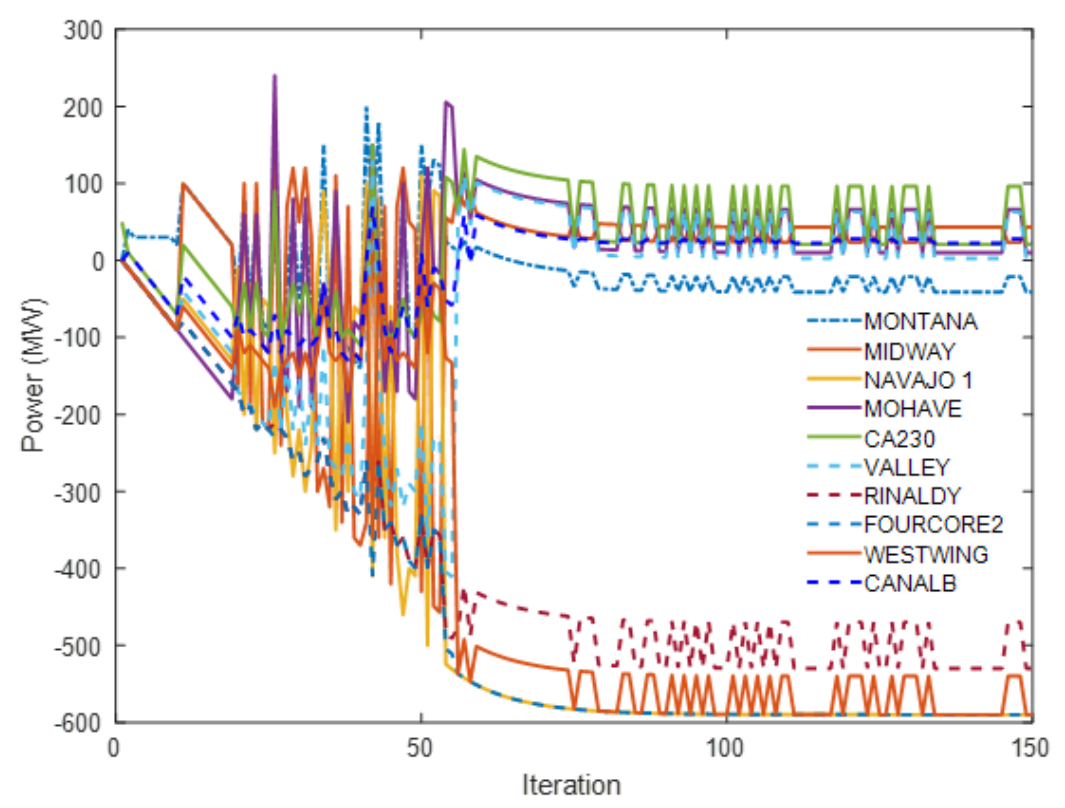

Fig. 4.2.: Active power versus iteration

Based on the results represented in Table 4.2, Figure 4.1, and Figure 4.2, min ISGA for the parameters of combination 7 is decreasing with a smooth behavior. Therefore, for the next step of this chapter, we are choosing the simulation time equals 12 second, 100 iterations, and step size 10. Figure 4.3 shows the generator rotor angle before applying control rules of combination 7 in Table 4.2. Figure 4.4 shows the generator angles after applying the control rules of combination 7. As it is shown in these figures, the control rules can reduce the generator angle differences.

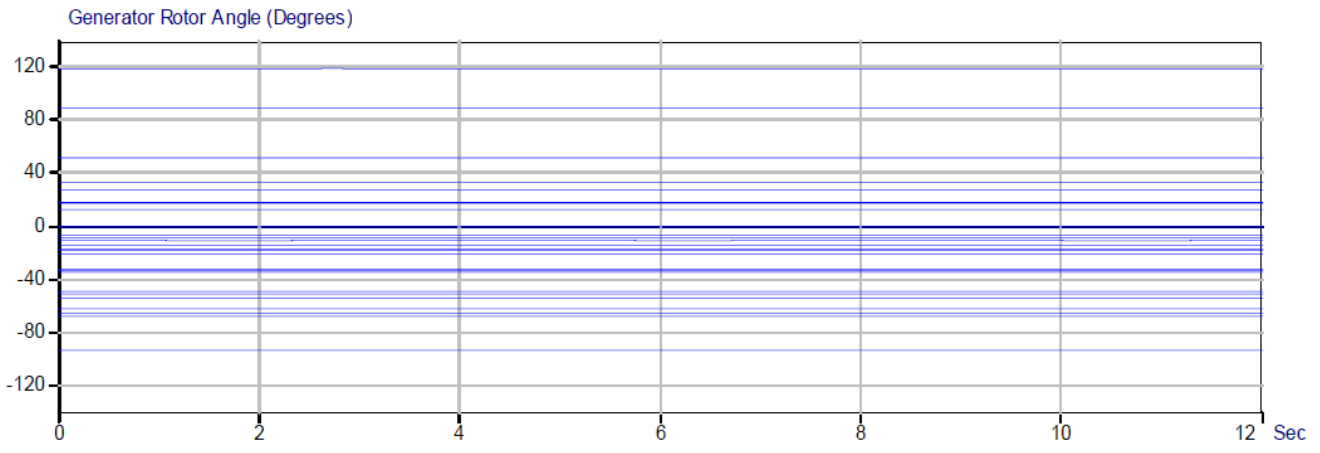

Fig. 4.3.: Generator rotor angles before applying control rules of combination 7 


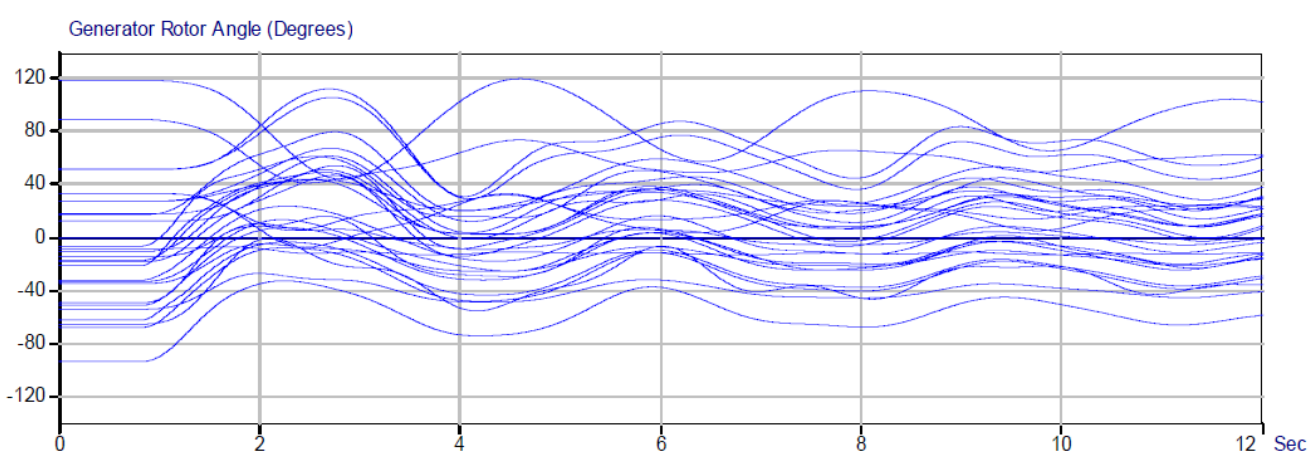

Fig. 4.4.: Generator rotor angles after applying control rules of combination 7

\subsubsection{Gradient Descent Result With the Presence of Event}

In this section, in order to evaluate the gradient descent algorithm, one event has been selected. This event is a Single Line to Ground (SLG) fault on the TABLE1 bus at $\mathrm{t}=0.6 \mathrm{~s}$, and it is clearing at $\mathrm{t}=0.67 \mathrm{~s}$. This event should have some properties. First, this event should be detected as an event, second, it needs to be controlled.

Table 4.3 shows seven different cases. In each case, we are seeking to find a control combination capable of stabilizing the event mentioned above. The algorithm stops whether the events stabilized or it reached the maximum number of iteration. The columns of Table 4.3 are the same parameters as the columns of Table 4.2.

Table 4.3.: Gradient Descant result with presence of an event

\begin{tabular}{|l|c|c|c|c|c|c|l|}
\hline & Jmin & alpha & It - dec & It & Ts & Initial & Active power for bus 1-10 \\
\hline 1 & 3.95 & 10 & $50-10$ & 150 & 12 & 50 & $10-110-30-110-30-90-110-110-70-30$ \\
\hline 2 & 7.9 & 10 & $10-5$ & 150 & 12 & 50 & $10.5-109.5-29.5-109.5-29.5-89.5-109.5-109.5-69.5-29.5$ \\
\hline 3 & 3.95 & 3 & $0-0$ & 150 & 12 & 50 & $6-108-48-108-42-54-108-108-72-36$ \\
\hline 4 & 3.95 & 1 & $100-10$ & 150 & 12 & 50 & $1.56-102.44-44.44-102.44-44.44-102.44-102.44-102.44-72.44-46.44$ \\
\hline 5 & 3.93 & 40 & $0-0$ & 160 & 12 & 50 & $1600-110-160160-160-160160-80$ \\
\hline 6 & 3.95 & 2 & $45-10$ & 160 & 12 & 50 & $2.19-105.81-37.81-105.81-49.81-105.81-105.81-105.81-77.81-53.81$ \\
\hline 7 & 3.95 & 3 & $45-10$ & 160 & 12 & 50 & $6-108-48-108-42-54-108-108-72-36$ \\
\hline
\end{tabular}




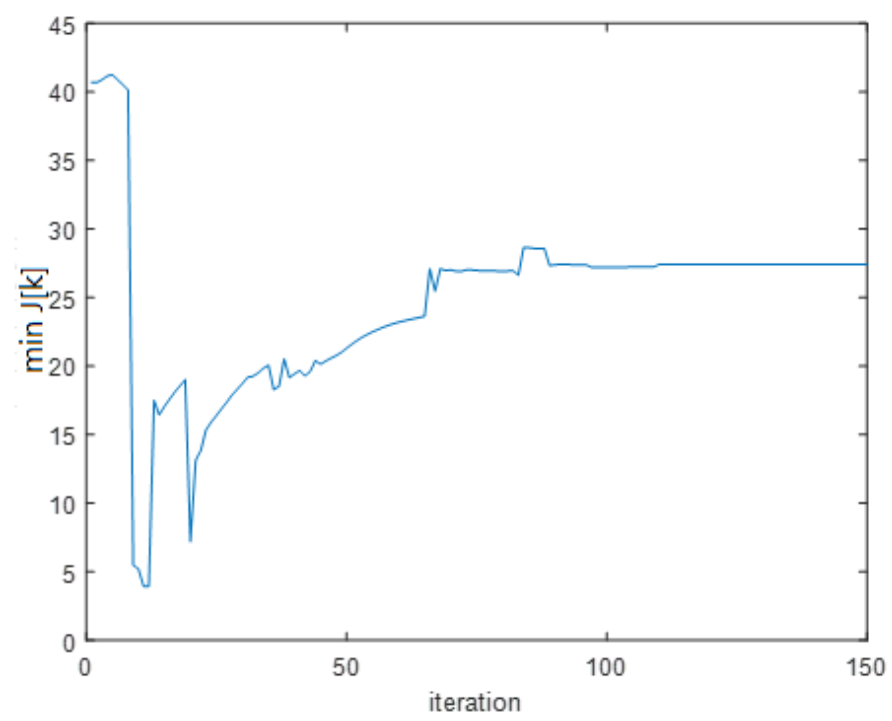

Fig. 4.5.: Minimum J[k] versus $\mathrm{k}$ for row 1 of Table 4.3

As it is represented for the result of combination 1 in Figure 4.3, the values that worked well without event does not return an acceptable result in combination 1 . In fact, the variations of Min ISGA is enormous, and it is not following a permissible pattern. One of the main issues related to this algorithm was it could not find a control combination that can stabilize the event. Actually, the algorithm continued until it reached to the maximum number of iteration, and it did not stop because the event was stabilized. Therefore, other optimization methods are applied in the next section for finding the best values of load shedding. One of these methods is Particle swarm optimization that is explained in the next section.

\subsection{Particle Swarm Optimization Method}

Particle Swarm Optimization (PSO) is basically an optimization technique for exploring the search space and minimizing/maximizing a particular objective [21]. The main idea starts with initiating a random population in the search space. Then the objective function is evaluated for every row of the initial matrix or agents, and 
the best value is selected among them. In this project, the objective function is ISGA, and the best value is the minimum ISGA. After finding the minimum ISGA, the rest of the agents are trying to move toward the location of the best agent.

One of the main difference of the PSO algorithm with a gradient descent algorithm is adding some random terms to this algorithm to increase the possibility of finding the correct solution and reducing the possibility of a local minimum. Another difference is a diverse random initial value initialized at the beginning of the algorithm. In each iteration of running the PSO algorithm, the population is updating by a velocity vector that can be calculated using the equation (4.5) [22]

$$
V_{i}(t+1)=\omega V_{i}(t)+c_{1} r_{1}\left[\hat{X}_{i}(t)-X_{i}(t)\right]+c_{2} r_{2}\left[g(t)-X_{i}(t)\right]
$$

The index of each particle at every iteration is represented by $i . V_{i}(t)$ is the velocity of particle $i$ at time $t$ and $X_{i}(t)$ is the position of particle $i$ at time $t . c_{1}$ and $c_{2}$ are two constant numbers between 0 and 2, and they are selected 2 in this research.

$r_{1}$ and $r_{2}$ are two random number between 0 and $1 . \hat{X}_{i}(t)$ is the best solution in each iteration. In this project, the solution related to the minimum ISGA in each iteration is selected as $\hat{X}_{i}(t) \cdot g(t)$ is the global best candidate solution up to the iteration t. $\omega$ is a parameter decreasing by increment of the number of iteration and it is calculated by $4.6[22]$.

$$
\omega=0.2+\frac{(0.9-0.2)}{(1-\text { maxiteration })}(\text { currentiteration }- \text { maxiteration })
$$

\subsubsection{The Result of PSO Algorithm For a Test Event}

We implemented the PSO algorithm related to our project. The objective function is ISGA. The maximum value of the load on each bus was selected $500 \mathrm{MW}$, and the minimum value was selected $-500 \mathrm{MW}$. The initial population matrix is evaluated randomly on the search space which is in the range $[-500,500]$ for each bus. This matrix has 50 rows; actually, it has $50 \times 10$ dimension. The maximum number of iteration is chosen 20 since usually the algorithm could return the solution in less than 6 iterations. 
Table 4.4 illustrates the result of PSO algorithm for seven different cases. Jmin is the minimum ISGA, which is the objective function of the algorithm. It includes two numbers: the first number is the iteration number that the algorithm stops, and the second number is the maximum iteration number. $T s$ is the simulation time, and the last column is the result of the best solution for the algorithm.

Table 4.4.: The result of PSO algorithm for 7 different cases

\begin{tabular}{|c|c|c|c|l|}
\hline & Jmin & It & Ts & Active power for bus 1-10 \\
\hline 1 & 3.93 & $4 / 20$ & $12 \mathrm{~s}$ & {$[95.23,-320.02,-130.01,-283.37,-55.20,-317.26,500,-333.91,416.71,500]$} \\
\hline 2 & 3.98 & $6 / 20$ & $12 \mathrm{~s}$ & {$[500,-500,285.21,-500,166.67,354.39,128.7,500,438.61,387.43]$} \\
\hline 3 & 3.925 & $2 / 20$ & $12 \mathrm{~s}$ & {$[-36-386-448-159392-160264-186-351-248]$} \\
\hline 4 & 3.90 & $5 / 20$ & $12 \mathrm{~s}$ & {$[500,225.14,500,-500,500,68.27,-500,-418.35,429.69]$} \\
\hline 5 & 3.937 & $2 / 20$ & $12 \mathrm{~s}$ & {$[200-432-426-14468-48615-13096229]$} \\
\hline 6 & 3.926 & $2 / 20$ & $12 \mathrm{~s}$ & {$[112-480-101-980-335270271-132486]$} \\
\hline 7 & 3.915 & $4 / 120$ & $12 \mathrm{~s}$ & {$[168.67,-500,271.67,-175.87,500,314.51,-255.97,-163.48,-198.03,-17.17]$} \\
\hline
\end{tabular}

According to Table 4.4, it can be seen that the PSO algorithm has some advantages and disadvantages in comparison to the gradient descent algorithm. One of the main advantages of PSO is it can always find a solution for the problem, and this solution can stabilize the event, while with gradient descent algorithm we could not find a solution leading to a stabilized event. Another advantage is its high speed for finding the solution since, after 2-6 iteration, it can return a solution leading to stabilizing the event. In the other hand, the main disadvantage of the PSO algorithm is the random initial population and random numbers in velocity calculation. Therefore, the solution is not unique in every run of the algorithm. In fact, as the Gradient descent also requires an initial starting point, its solution is not unique. 


\subsubsection{The Result of the PSO algorithm for a Set of Events}

In this section, a set of events including 4803 -phase events has been selected to find a proper control for any of them using the PSO algorithm. The algorithm is only applied to 100 events. Therefore, a separate control combination is found regarding to any of the events [13].

In this section, to stabilize more events, the search space is expanded. So, 27 buses have been selected instead of 10 buses. These 27 buses have a load equal to $500 \mathrm{MW}$ or higher on them. The number of particles in the PSO algorithm is also selected as 50. Therefore, the search space is a $50 \times 27$ dimension. Table 4.5 represents these 27 buses.

Table 4.5.: 27 buses where control is applied using the PSO algorithm

\begin{tabular}{|l|l|l|l|l|l|l|}
\hline MONTANA 500 & MIDWAY 200 & CA230 230 & HANFORD 500 & SAN JUAN 345 & WESTWING 500 & CANADA 500 \\
\hline PALOVRDE 500 & TEVATR 500 & NORTH 500 & JOHN DAY 500 & LITEHIPE 230 & CELILO 230 & PARDEE 230 \\
\hline MIRALOMA 500 & CRAIG 345 & INTERMT 345 & CORONADO 500 & SERRANO 500 & VINCENT 230 & STA J 230 \\
\hline STA E 230 & ELDORADO 500 & TEVATR 200 & DEVERS 500 & MIDPOINT 345 & CAMP WIL 345 & \\
\hline
\end{tabular}

After applying PSO algorithm for 100 unstable events, 100 various control combinations are found. From these 100 events, 42 of them can be stabilized by the control combination found by PSO. Table 4.6 represents the results of the PSO algorithm for 8 sample events that became stable using the new control combinations found by PSO. These events remained unstable after applying the control combinations used in Chapter 3 and in $[2,15]$.

In Table 4.6, Event is the event number. gbest is the global best solution in the PSO algorithm, $C_{1}, C_{2}$ are the constant values in the equation (4.5) and the last column is the best solution of PSO algorithm that stabilizes the unstable event.

Figure 4.6 and Figure 4.7 show the generator rotor angle for the event number 166 before and after controlling. Event number 166 is a 3-phase fault on the line between MALIN7 and MALIN8. As it is shown in these figures, control combination found by PSO could effectively reduce the generator rotor angle differences. 
Table 4.6.: PSO results for 8 sample events

\begin{tabular}{|c|c|c|c|c|}
\hline & Event & gbest & $C_{1}, C_{2}$ & Active Power of buses $1-27$ \\
\hline 1 & 143 & 3.95 & 3 & $\begin{array}{l}500-483.47164 .67-103.67-68.67282 .33 \quad 339.33-403.76 \\
-350.33293 .33-478.33-500-144.33-295.33137 .19-163.67-117.07-319.33 \\
-123.41-147.6020 .33109 .67136 .67-122.48-500181.33-282.33\end{array}$ \\
\hline 2 & 166 & 3.91 & 3 & $\begin{array}{l}234.33-245.33184 .3369 .6751 .33-115.378263 .54-185.33-346.33-74.33 \\
-60.67-411.33-170.21100 .67-17.67199 .34140 .33-500-30.33 \\
-218.33187 .33-142.33154 .3333-277.33-59.67235 .33208 .33\end{array}$ \\
\hline 3 & 183 & 3.93 & 3 & $\begin{array}{l}381.94-171.647500113-38-500139.06166 .67-42.49 \\
458.07-338.67-174.5275 .8402 .67-18-166.67-122.42-500292.67 \\
184.6739-488.67-500-260.67213 .83-244.666746 .30\end{array}$ \\
\hline 4 & 302 & 3.9 & 2 & $\begin{array}{l}162.67-20.33319 .84-141.67-92.1149 .67-137.55309 .76 \\
-76.67432 .33175 .33-50.33-379.3384 .33-144.8341 .33-39.33 \\
43.67-500-115.40-176.83-33.53-500-123.33-219.22-500-422.33\end{array}$ \\
\hline 5 & 321 & 3.93 & 2 & $\begin{array}{l}-131.67-194.3365 .33-95.33-187.56158 .67-250.33123 .6710 .673 .67 \\
-40.6796 .6797 .33-488.33-10.67200 .64-337.33233 .6-70.67-278.33-163.67 \\
-72.67-108.67-152.33-490.33164 .67-36.67\end{array}$ \\
\hline 6 & 342 & 3.96 & 3 & $\begin{array}{l}160.67-111.33403 .96-363.33-162.838 .67138 .67-134.6763 .67 \\
395.12-281.33113 .67-500-422.33-93.67-124.34260 .33-497.2717 .67 \\
-148.67125 .77-154.33-148.67-182.33-486.33-276.33-162.76\end{array}$ \\
\hline 7 & 374 & 3.90 & 3 & $\begin{array}{l}466.33-486.3362 .67-254.33-88.33224 .76290 .33-20.67-117.33 \\
500.0076 .67-418.33-500-133.67-377.33313 .55211 .33 \\
97.67-101.67-365.33-115.67-271.33500-283.33138 .67-33.3327 .33\end{array}$ \\
\hline 8 & 382 & 3.93 & 3 & $\begin{array}{l}500-500500-10.97-64.78500 .0093 .99-85.41411 .94 \\
500-432.33500-107.85-279.11-500-65.8500382 .33 \\
251.28-500-88.60-500-500-500-500-326.94-500\end{array}$ \\
\hline
\end{tabular}




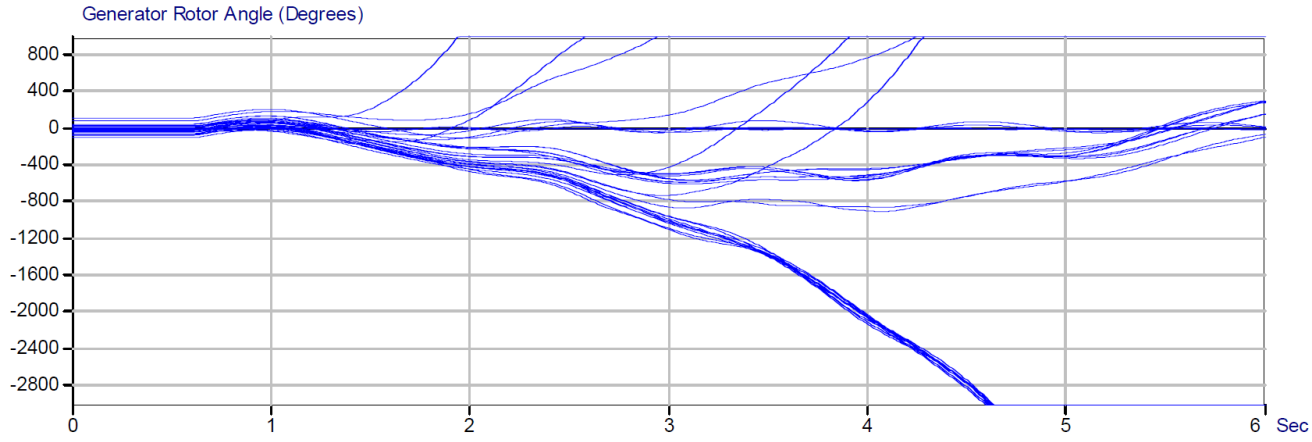

Fig. 4.6.: Generator rotor angles during a 3 phase event before applying any control.

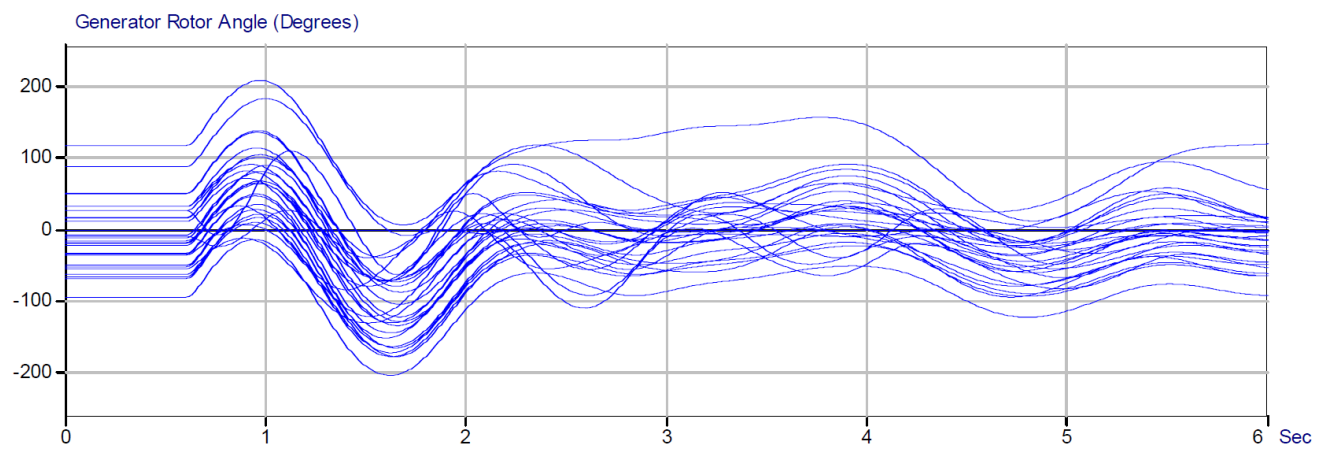

Fig. 4.7.: Generator rotor angles during a 3 phase event after applying the control found by the PSO algorithm. 


\section{DECISION TREES FOR CONTROL SELECTION}

\subsection{Overview}

The main idea of this chapter is to find an algorithm that can select from different control combinations for stabilizing various events. According to the results of the previous chapters, the events stabilized by each of the control sets were not all in common. So the number of stabilized events can be increased if we could use a method that can choose between different control combinations. In fact, different artificial intelligence methods can be employed for this purpose, like Neural Network $(\mathrm{NN})$, and Decision Trees (DTs).

According to previous studies, the same fixed control combination is applied to every event [1-3]. This thesis applies different control combinations to different events.

\subsection{Control combinations}

Using the set of buses for control in Chapter 3, and using the optimization results from Chapter 4, we tested a method that applies one of the three control combinations listed below.

- Old: the control combination found in Chapter 4

- 382: PSO result for the event 382

- 166: PSO result for the event 166

The reason for selecting the control combination found for event 382 and event 166 is that they can stabilize more events than the rest of the control combination. Control combination Old is similar to $500 \mathrm{MW}$ fast power increases on two buses 
(MONTANA and CA230) and reducing the same amount of load on three other buses MIDWAY, NAVAJO, and MOHAVE. Table 5.1 illustrates the details of three control sets considered in this section.

These controls can reduce angle differences in the AC network [2]. The process of selecting control sets is done through Machine Learning algorithms.

\subsection{Data sets}

Our classification model for DT is created offline from the training data set where each data point consists of an input vector along with a target value, which shows the class of that sample. Our data set is simulated using 1345 discrete events on the 176-bus model.

The training set includes data from 385 six-second simulations. Each event is considered as an independent case that is simulated during 6 seconds. The events include short circuit to ground faults on 40 transmission lines in the WECC model. The test set includes 960 events containing 480 1-phase short circuit faults and 480 3-phase short circuit faults.

To obtain the data sets, TSAT software is used in combination with MATLAB for creating the power flow. For every event included in the training or test sets, in each time step, TSAT software provides generator voltage angles and magnitude, and bus voltage angles and magnitude recorded by 17 PMUs. Then bus frequencies, bus magnitude variation, ISBA, and the derivative of the ISBAs, etc. are calculating. Therefore, using 17 PMUs measurement data, finally, we have 77 features.

Based on the stability condition applied in [2], an event is unstable if it has a maximum generator angle difference greater than 300 degrees.

To record the data, after detecting an event, 4 cycles are allowed for the event to be over, and then 5 sample points are collected. The 4 cycles were evaluated based on a trial and error method in [15]. The next step is to determine the target value for each sample. Every control combination has the ability to stabilize a set 
Table 5.1.: The detail of three control combinations

\begin{tabular}{|c|c|c|c|c|c|}
\hline \multicolumn{2}{|c|}{ Control Combination 1} & \multicolumn{2}{|c|}{ Control combination 2} & \multicolumn{2}{|c|}{ Control Combination 3} \\
\hline Bus Name & Power (MW) & Bus Name & Power (MW) & Bus Name & Power (MW) \\
\hline & & MONTANA & 500 & MONTANA & 234.33 \\
\hline & & MIDWAY & -500 & MIDWAY & -245.33 \\
\hline & & CA230 & 500 & CA230 & 184.33 \\
\hline & & HANFORD & -10.97 & HANFORD & 69.67 \\
\hline & & SAN JUAN & -64.78 & SAN JUAN & 51.33 \\
\hline & & SAN JUAN & 500 & SAN JUAN & -115.38 \\
\hline & & CANADA & 93.99 & CANADA & 263.54 \\
\hline & & PALOVRDE & -85.41 & PALOVRDE & -185.33 \\
\hline & & TEVATR & 411.94 & TEVATR & -346.33 \\
\hline & & NORTH & 500 & NORTH & -74.33 \\
\hline & & JOHN DAY & -432.33 & JOHN DAY & -60.67 \\
\hline MONTANA & 500 & LITEHIPE & 500 & LITEHIPE & -411.33 \\
\hline MIDWAY & -500 & CELILO & -107.85 & CELILO & -170.21 \\
\hline NAVAJO1 & -500 & PARDEE & -279.11 & PARDEE & 100.67 \\
\hline MOHAVE 500 & -500 & MIRALOMA & -500 & MIRALOMA & -17.67 \\
\hline CA230 & 500 & CRAIG & -65.8 & CRAIG & 199.34 \\
\hline & & INTERMT & 500 & INTERMT & 140.33 \\
\hline & & CORONADO & 382.33 & CORONADO & -500 \\
\hline & & SERRANO & 251.28 & SERRANO & -30.33 \\
\hline & & VINCENT & -500 & VINCENT & -218.33 \\
\hline & & STA J & -88.6 & STA J & 187.33 \\
\hline & & STA E & -500 & STA E & -142.33 \\
\hline & & ELDORADO & -500 & ELDORADO & 154.33 \\
\hline & & TEVATR & -500 & TEVATR & -277.33 \\
\hline & & DEVERS & -500 & DEVERS & -59.67 \\
\hline & & MIDPOINT & -326.94 & MIDPOINT & 235.33 \\
\hline & & CAMP WIL & -500 & CAMP WIL & 208.33 \\
\hline
\end{tabular}


of events. Hence, in the first step, we have to find three data sets associated with every control combinations. Therefore, the target value is evaluated for each of the control combinations separately. Every data set categorizes the events into stable and unstable. The target value is Boolean; 1 is for stable, and 0 is for unstable. If the control combination can stabilize the event, the target is assigned 1, otherwise, the target value is 0 . We did the simulation for all the control combinations and assigned each sample with the proper target value, and three training data sets is recorded for each control combination.

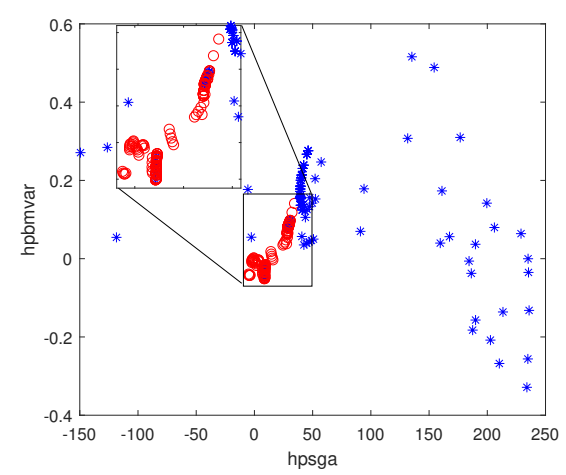

(a) Control combination 1 .

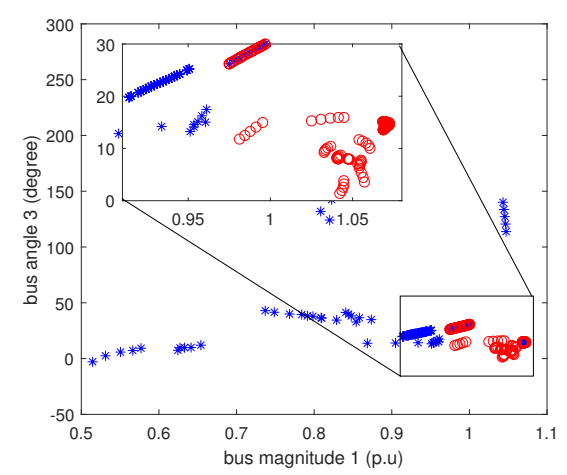

(b) Control combination 2 .

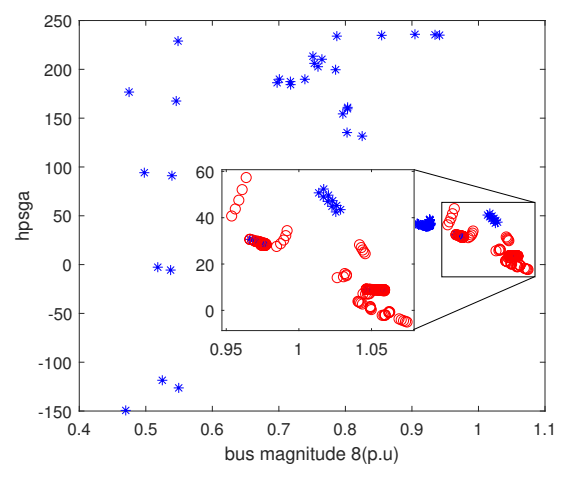

(c) Control combination 3 .

Fig. 5.1.: Training data. 
The next step is to determine the target value for each sample. Every control combination has the ability to stabilize a set of events. Therefore, three data sets associated with every control combinations should be generated. Every data set categorizes the events into stable and unstable. The target value is Boolean; 1 is for stable, and 0 is for unstable. If the control combination can stabilize the event, the target is assigned 1 , otherwise, the target value is 0 .

The features related to each sample data is recorded before applying the control combination. Hence, the features for each sample is the same for all the three data sets. The target value is evaluated after applying the control combination; if the event can be stabilized by the control combination, the target value for the corresponding sample data is 1 , otherwise, the target value is 0 . Therefore, the target values regarding each sample data are the only difference between the three control sets, and it is evaluated for every control combinations separately.

Figure 5.1, shows the scattering of training data set for two features of the samples. Figure $5.1 \mathrm{a}, \mathrm{b}$, and c shows the training data for control combination 1, 2, and 3 respectively. These features are selected based on the training results of the DTs in the next section, and they are different for each control combination.

Test data is also visualized using the same method for training data. Figure 5.2 represents the scattering plot for two features related to Test data for control combination 1,2 , and 3 .

\subsection{Algorithm}

The simulation is carried out using Machine Learning algorithms. Hence, Decision Trees (DTs) and neural networks both are practical solutions to solve this problem $[1-4,6]$. 


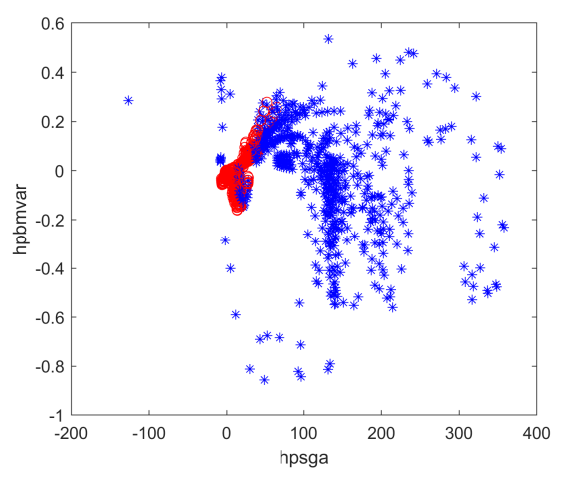

(a) Control combination 1.

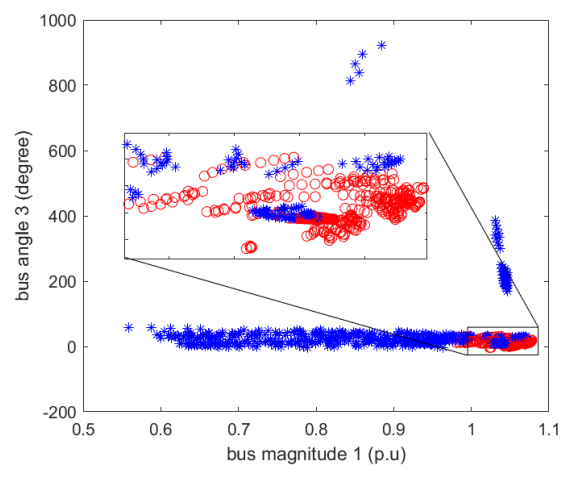

(b) Control combination 2 .

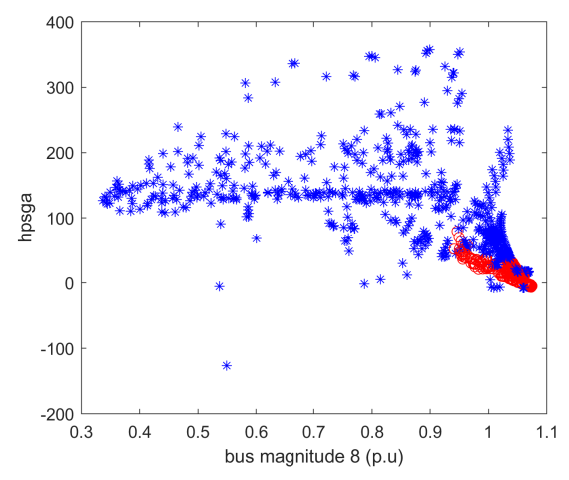

(c) Control combination 3 .

Fig. 5.2.: Testing data.

In this project, DTs are selected for the classification method. The main advantage of DTs over other pattern recognition tools is the training time. Another advantage is that with a large number of predictor variables available, a small subset of these variables is normally used in the trained DT. Therefore, DTs are more resilient to missing data [19].

To begin, the training data sets are selected for each of control combinations, and a separate DT is developed related to each control combination. Then a strategy is exploited to select the best control combination for any of the events. As mentioned in the previous sections, these three training data sets are generated from the same events but using a different control. It means, the training for two control combina- 
tions is done separately and independently or for each of the control combinations. A separate training data test is recorded, and in each of these data sets, two class of $1 / 0$ or stable and unstable have existed.

After completing the training data, a test set including 960 events is selected. For this test data set, the data is recorded in a similar way to the training data set. It means, 5 sample points are recorded after event detection.

Finally, by comparing the result of three algorithms for each event, we can select the control combination that has the ability to stabilize more sample points of each event.

\subsection{Implementation of the method}

Basically, our problem for training the algorithm of each control combinations is a Boolean classification problem since the output is stable/unstable or $1 / 0$. Every node in the decision tree can be represented by a variable or a feature. Eventually, the leaf nodes show the target value of the input vectors. In order to minimize the depth of the final tree, a greedy approach is planned to be used in this project [23]. Using a cost function, the different split points are tried and tested. We have used a Gini cost function since it performs well with noisy data set. The basic concept of Gini cost function is to search for the largest class in the training data set and isolate it from the rest of the data [8]. In fact, this function represents the purity of the nodes. Figure 5.3 shows an example of the decision tree [24].

The classification algorithm used in this study consists of several steps. The root node receives the entire training data set as input. Usually, all nodes are asking a true-false question about one of the features. Two types of question can be asked based on the type of features in the data set greater equal $>=$ or less equal $<=$. Greater equal $>=$ is used for the questions asked in this project. In response to this question, the data set is split or divided into two subsets. The new subsets are the input to the two child nodes. The goal of the questions is dividing the labels as far 


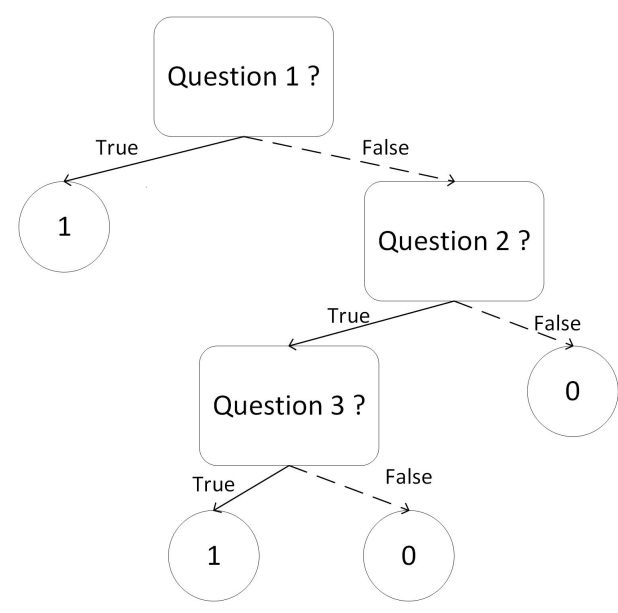

Fig. 5.3.: A sample decision tree.

as possible. The tree is proceeding down to find the purest possible distribution of the labels at each node, or when there is no uncertainty about the type of the label. In order to quantify how much a question unmixed the labels a metric called Gini impurity is used in the current project [25]. In order to quantify how much a question reduces the amount of uncertainty, a concept called information gain was used. There are many types of equations for calculating the Gini impurity and information gain. In this project, 5.1 shows the Gini function, and equation 5.2 shows the Impurity gain.

$$
\begin{gathered}
G i n i=1-\sum_{i} P_{i}^{2} \\
\left.I G=C U-P_{\text {left }} * G i n i(\text { Left })-P_{\text {right }} * \text { Gini(right }\right)
\end{gathered}
$$

In 5.1, $P_{i}$ is the probability of the labels. As we only have two labels, the maximum of $i$ is equal to 2 . In 5.2, $I G$ shows the information gain, $C U$ shows the current uncertainty, $P_{\text {left }}$ and $P_{\text {right }}$ show the probability of the left and right node respectively in each iteration. Current uncertainty in the root node equals the Gini impurity of that node and as the tree proceed the uncertainty of the DT in each iteration is calculated using 5.2. 
Using Gini impurity function and information gain, the best question can be selected at each node. Then we continue recursively to build the tree on each of the new nodes. The data is continuously dividing until there is no question to ask. Figure 5.4 shows the decision tree learning algorithm. The detail of some important functions which are used in training of the DT are represented in Appendix A.

The main issue related to DT is over-fitting. To defeat the over-fitting issue, pruning methods can be used such as defining a threshold for the number of observations in a node. Another method can be early stopping [26]. One of the effective methods to avoid over-fitting in DT is the random forest method. In the random forest, a random data set from the training data is selected, and a separate DT is training accordingly. Then an integration method can be applied to find the output. Finally using these methods over-fitting can be reduced.

\subsection{Result}

As mentioned in Chapter 1, the test system used in this research is a simplified model of Transmission lines the Western Electricity Coordinating Council (WECC) including 29 machines and 176 buses as illustrated in Figure 1.1.

For recording the training set, a file consisting of 385 disturbances is used. This file includes four single outage contingencies for each of 40 transmission lines. Moreover, 210 double outage contingencies involving two lines plus 15 additional single contingency. Every event is simulated during 6 seconds by TSAT. The parameters such as voltage magnitudes, voltage angles and etc. are measuring through 17 PMUs. For each event, an event detection algorithm is applied explained in [13], and the eligibility for control is investigated. The detail of the procedure for data recording and employed features are explained in Section 5.3. 


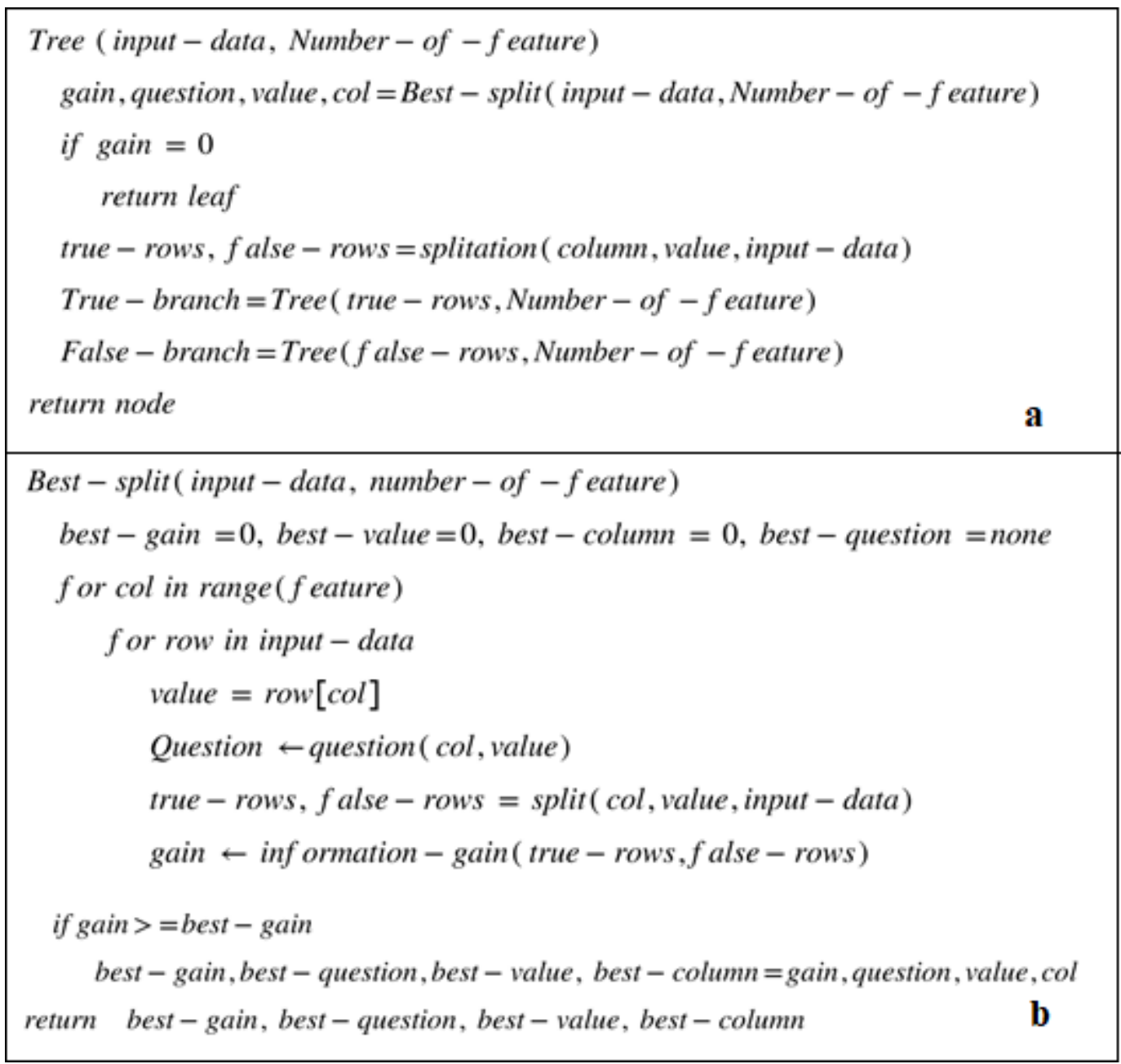

Fig. 5.4.: The decision tree learning algorithm. a) this function shows the main procedure for building the tree b) this function is finding the best value and column for a data set

Two methods have been used for training the DTs; the first is a program written in Python and the second is MATLAB DT function. Both methods are based on the CART algorithm. Three different DTs are trained by both methods, and the visual representation of the trees are shown in Appendix A.

The visual representation of the DTs for the control combination 1, control combination 2, and control combination 3 are illustrated in Figure A.1, Figure A.2, and Figure A.3 of Appendix A respectively. In these figures, all the decision nodes are 
represented by a question, which is the best question based on the information gain in each level of the DT. The straight lines show the true answers in the right and the false answers on the left-hand side. The leaf nodes are represented by a number, which is the label allocated to that node. As we can see in these figures, in each node the best feature was selected, for example, bus magnitude $1>=0.968$ for the root node in the top of the tree in Figure A.2, and bus magnitude $8>=0.947$ for the root node at the top of the tree in Figure A.3.

The depth of the tree in Figure A.1 is 12 and for DT 1 and for the DT 2 in Figure A.2 is 8 . In Figure A, the depth of DT 3 is 9 , which make the DTs very complex. In order to test the DT model, the testing data set including 1045 samples are used. The results for the accuracy of both methods are shown in Table 5.2. Each sample is classified by applying the rules of the DT, and the target 1 or 0 is assigned to that. Next, the label is compared with the correct label which is determined from the simulation result. The accuracy was evaluated by counting the number of samples that correctly classified divided by the whole existing training samples.

In Table 5.2, PY, MAT show the results of Python code and MATLAB function respectively. As it is shown in this table, the DT reach to a high accuracy; approximately 90 percent for all the control combinations. Although they returned different DTs, similar results for accuracy of test and train data is evaluated, as represented in Table 5.2.

Because of the depth of the DT, to completely implement them in our algorithm, an easy approach is to load the DTs evaluated by MATLAB, and use "predict" function to estimate the label of every new sample.

In the next step, for each event that is detected by the method in [13], five samples after the fault end are processed by DT1, DT2 and DT3. The output value 1 means the event is predicted to be stabilized by the control. A score is calculated for each DT by adding the five output values for each DT to obtain a number between $0-5$. The control with the largest score is applied to the event. If all the scores are equal, for example 5,5,5, the control combination 1 is selected. 
Table 5.2.: The Accuracy results for learning and testing the DTs using a Python code and MATLAB libraries.

\begin{tabular}{|c|l|l|l|l|}
\hline Control combination & PY. Test (\%) & PY. Train (\%) & MAT. Test (\%) & MAT Train (\%) \\
\hline 1 & 88.71 & 93.94 & 92.15 & 93.64 \\
\hline 2 & 86.22 & 89.39 & 89.76 & 89.39 \\
\hline 3 & 93.94 & 93.59 & 86.79 & 93.94 \\
\hline
\end{tabular}

Table 5.3 illustrates the result after applying the new algorithm for the 960 events. The columns of this table show respectively the number of events controlled, the number of events unnecessary to control, the number of events stabilized after applying the control, average control time, and success rate of the algorithm.

Table 5.3.: Performance of 3 DTs for control selection 1 phase and 3 phase faults.

\begin{tabular}{|l|l|l|l|l|l|}
\hline Test set & Controlled & Unnecessary & Stabilized & Tavg & Success rate \\
\hline 1 phase & 96 & 6 & 36 & 0.77 & 0.375 \\
\hline 3 phase & 113 & 13 & 36 & 0.91 & 0.318 \\
\hline Total & 209 & 19 & 72 & 0.84 & 0.3465 \\
\hline
\end{tabular}

Table 5.4.: Performance of 1 DT for control selection 1 phase and 3 phase faults [13].

\begin{tabular}{|l|l|l|l|l|l|}
\hline Test set & Controlled & Unnecessary & Stabilized & Tavg & Success rate \\
\hline 1 phase & 96 & 6 & 21 & 0.77 & 0.219 \\
\hline 3 phase & 112 & 13 & 28 & 0.91 & 0.250 \\
\hline Total & 208 & 19 & 49 & 0.85 & 0.236 \\
\hline
\end{tabular}


By comparing the results of Table 5.3 by the results in Table 5.4 in [13] for the same test set, the number of stabilized events is 49 , and the success rate is 0.236 . Therefore, using the algorithm with control selection could improve the number of the stabilized event by $32 \%$, and success rate by $31 \%$.

Figure 5.5 shows the generator rotor angles for 29 generators of the model during a transient event for 6 seconds. A 3 phase fault occurred at 0.55 second on the line between Hanford and John day buses and cleared at 0.67 seconds. Figure 5.6 shows the simulation of generator rotor angles for the same fault after applying the algorithm. This event is stabilized by control combination 2. The algorithm can effectively identify the appropriate control combination and stabilize it using 3 DTs.

Figure 5.7 shows the generator rotor angles for 29 generators of the model during a transient event simulated for 6 seconds. This fault is also a 3 phase fault occurred at 0.52 second on the line between Table 1 and Table 2 buses, and cleared at 0.67 seconds. Figure 5.8 plotted generator rotor angles for the same transient event after applying the algorithm. This event is stabilized by control combination 3. The algorithm has the ability to identified different events and categorized the proper control based on their features.

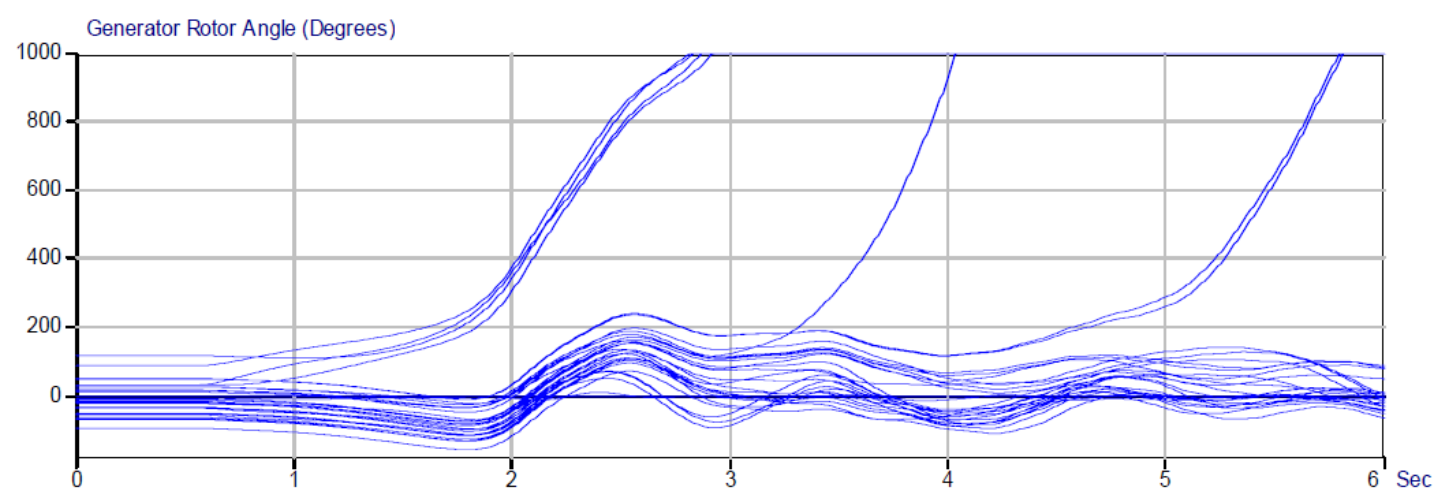

Fig. 5.5.: Generator rotor angles during a 3 phase event before applying the control selection algorithm. 


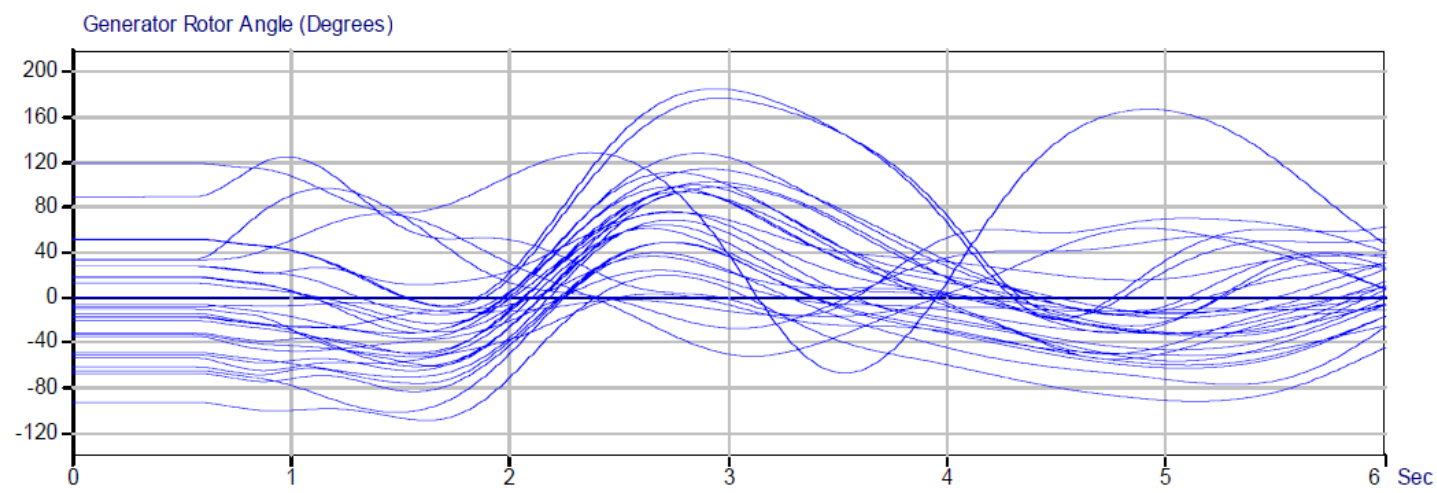

Fig. 5.6.: Generator rotor angles during a 3 phase event after applying the control selection algorithm and selecting control combination 2 .

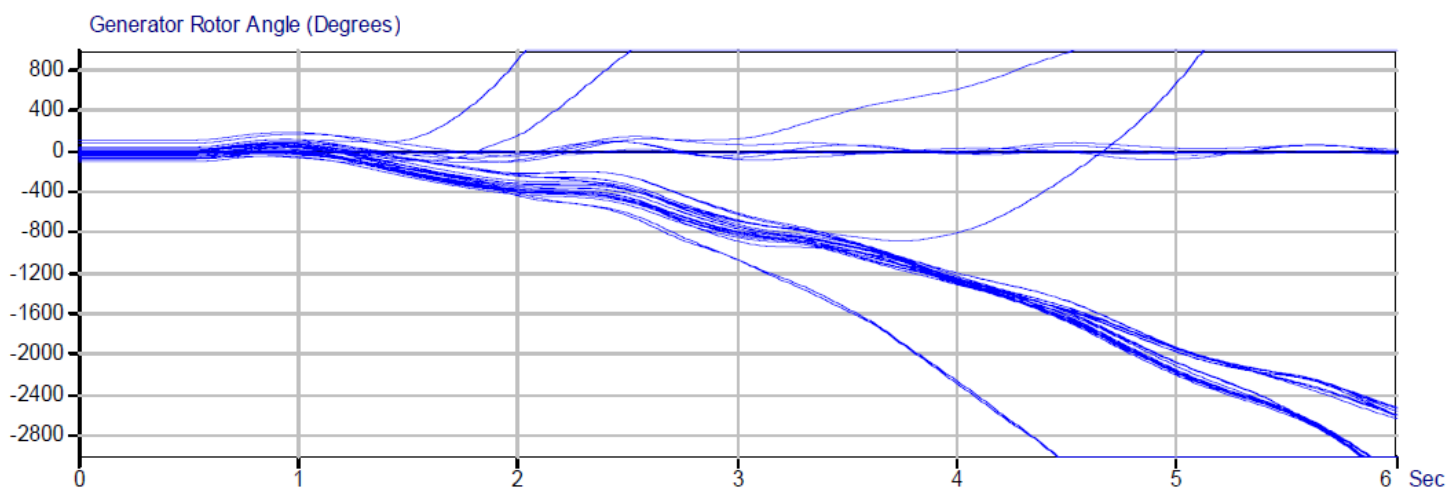

Fig. 5.7.: Generator rotor angles during a 3 phase event before applying the control selection algorithm. 


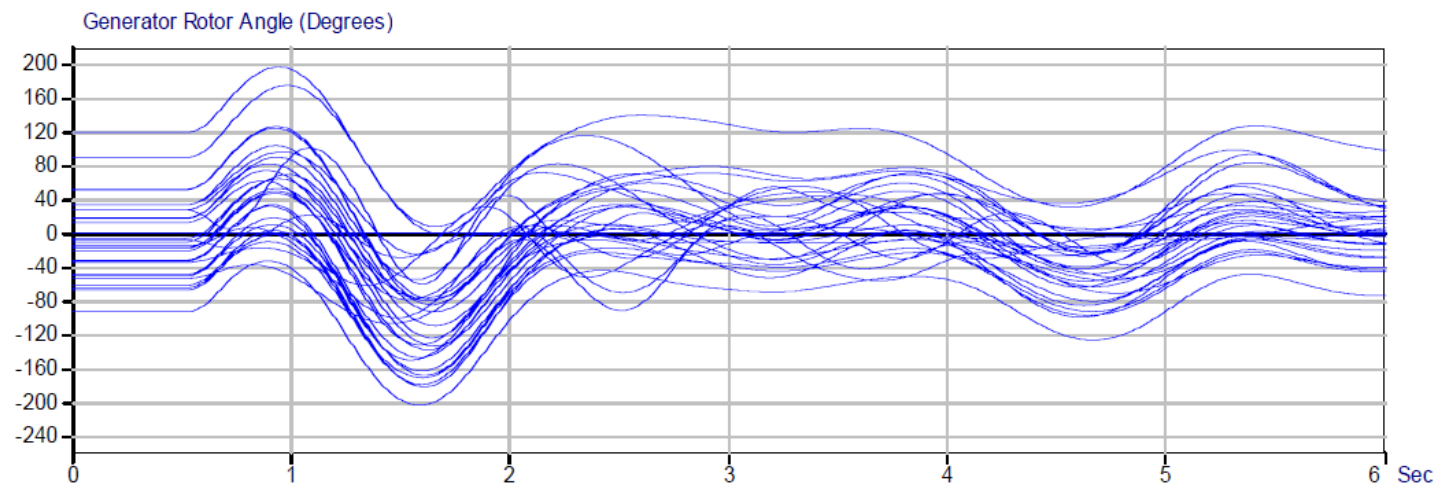

Fig. 5.8.: Generator rotor angles during a 3 phase event after applying the control selection algorithm and selecting control combination 3 . 


\section{CONCLUSION}

The one-shot control method is an effective way to stabilize electrical disturbances in the electrical transmission system. This method modifies the amount of load in some specific locations of the power system to control and stabilize the events. Determining the amount of the loads and the location of load variation is crucial.

In this project, using the sensitivity analysis, the most effective buses are located in the power system. In the next step, the Particle Swarm Optimization (PSO) algorithm is used to increase the effectiveness of the one-shot control scheme by determining the amount of load shed or added on the buses. The sensitivity analysis and PSO algorithm lead to different control combinations. Every control combination can stabilize different types of events. In the final step of this project, for three control combinations that were found by PSO, three Decision Trees (DT) are trained. An algorithm is developed with the ability to decide between the three control combinations. The final results showed the algorithm stabilizes 72 events out of 209 events that have control action, which is $30 \%$ improvement in comparison to the previous result. 
REFERENCES 


\section{REFERENCES}

[1] K. Mei and S. M. Rovnyak, "Response-based decision trees to trigger one-shot stabilizing control," IEEE Transactions on Power Systems, vol. 19, no. 1, pp. 531-537, 2004.

[2] Q. Gao and S. M. Rovnyak, "Decision trees using synchronized phasor measurements for wide-area response-based control," IEEE Transactions on Power Systems, vol. 26, no. 2, pp. 855-861, 2011.

[3] S. M. Rovnyak, C. W. Taylor, and Y. Sheng, "Decision trees using apparent resistance to detect impending loss of synchronism," IEEE Transactions on Power Delivery, vol. 15, no. 4, pp. 1157-1162, 2000.

[4] Y. Sheng and S. M. Rovnyak, "Decision tree-based methodology for high impedance fault detection," IEEE Transactions on Power Delivery, vol. 19, no. 2, pp. 533-536, 2004.

[5] M. Mishra and P. K. Rout, "Detection and classification of micro-grid faults based on hht and machine learning techniques," IET Generation, Transmission \& Distribution, vol. 12, no. 2, pp. 388-397, 2017.

[6] A. Khamis, Y. Xu, Z. Y. Dong, and R. Zhang, "Faster detection of microgrid islanding events using an adaptive ensemble classifier," IEEE Transactions on Smart Grid, vol. 9, no. 3, pp. 1889-1899, 2018.

[7] F. Hashemi, M. Mohammadi, and A. Kargarian, "Islanding detection method for microgrid based on extracted features from differential transient rate of change of frequency," IET Generation, Transmission \&6 Distribution, vol. 11, no. 4, pp. 891-904, 2017.

[8] N. W. A. Lidula and A. D. Rajapakse, "A pattern-recognition approach for detecting power islands using transient signalspart ii: Performance evaluation," IEEE Transactions on Power Delivery, vol. 27, no. 3, pp. 1071-1080, 2012.

[9] L. Zheng, W. Hu, K. Hou, X. Xu, and G. Shao, "Real-time transient stability assessment based on deep recurrent neural network," in Innovative Smart Grid Technologies-Asia (ISGT-Asia), 2017 IEEE. IEEE, 2017, pp. 1-5.

[10] R. Diao, V. Vittal, K. Sun, S. Kolluri, S. Mandal, and F. Galvan, "Decision tree assisted controlled islanding for preventing cascading events," in Power Systems Conference and Exposition, 2009. PSCE'09. IEEE/PES. IEEE, 2009, pp. 1-8.

[11] L. Ding, Y. Guo, P. Wall, K. Sun, and V. Terzija, "Identifying the timing of controlled islanding using a controlling uep based method," IEEE Transactions on Power Systems, vol. 33, no. 6, pp. 5913-5922, 2018. 
[12] R. J. Best, D. J. Morrow, D. M. Laverty, and P. A. Crossley, "Techniques for multiple-set synchronous islanding control," IEEE Transactions on Smart Grid, vol. 2, no. 1, pp. 60-67, 2011.

[13] S. M. Rovnyak, D. W. Longbottom, D. C. Vasquez, and M. N. Nilchi, Monitoring and Control using Synchrophasors in Power Systems with Renewables. IET, 2017, ch. Response-Based Event Detection for One Shot Wide-Area Stability Controls.

[14] T. Shekari, A. Gholami, F. Aminifar, and M. Sanaye-Pasand, "An adaptive widearea load shedding scheme incorporating power system real-time limitations," IEEE Systems Journal, vol. 12, no. 1, pp. 759-767, 2018.

[15] M. Naghsh Nilchi, "Electric utility planning methods for the design of one shot stability controls," MSECE Thesis, Purdue University, IUPUI, 2013.

[16] wikipedia. (2018) Wecc intertie paths. [Online; accessed 26-March-2019]. [Online]. Available: https://en.wikipedia.org/wiki/WECC Intertie Paths

[17] TSAT: Transient Security Assessment Tool (User Manual), Power Tech Labs Inc., April 2018.

[18] PSAT: Powerflow Short-circuit Analysis Tool (User Manual), Power Tech Labs Inc., April 2011.

[19] G. Li and S. M. Rovnyak, "Integral square generator angle index for stability ranking and control," IEEE Transactions on Power Systems, vol. 20, no. 2, pp. 926-934, 2005.

[20] S. M. Rovnyak, G. Li, and K. Mei, "One-shot controls for preventing loss of synchronism," in 2003 IEEE Power Engineering Society General Meeting (IEEE Cat. No. 03CH37491), vol. 4. IEEE, 2003, pp. 2045-2052.

[21] J. Kennedy, "Particle swarm optimization," Encyclopedia of machine learning, pp. 760-766, 2010.

[22] M. E. H. Pedersen, "Good parameters for particle swarm optimization," Hvass Lab., Copenhagen, Denmark, Tech. Rep. HL1001, 2010.

[23] S. J. Russell and P. Norvig, "Artificial intelligence: a modern approach. malaysia," Pearson Education Limited. Rycroft-Malone, J.(2004). The PARIHS frameworkA framework for guiding the implementation of evidence-based practice. Journal of nursing care quality, vol. 19, no. 4, pp. 297-304, 2016.

[24] L. Breiman, Classification and regression trees. Routledge, 2017.

[25] A. Géron, Hands-on machine learning with Scikit-Learn and TensorFlow: concepts, tools, and techniques to build intelligent systems. "O'Reilly Media, Inc.", 2017.

[26] F. Esposito, D. Malerba, and G. Semeraro, "A comparative analysis of methods for pruning decision trees," IEEE Transactions on Pattern Analysis 86 Machine Intelligence, no. 5, pp. 476-491, 1997. 
APPENDIX 


\section{A. DECISION TREES VISUAL REPRESENTATION}

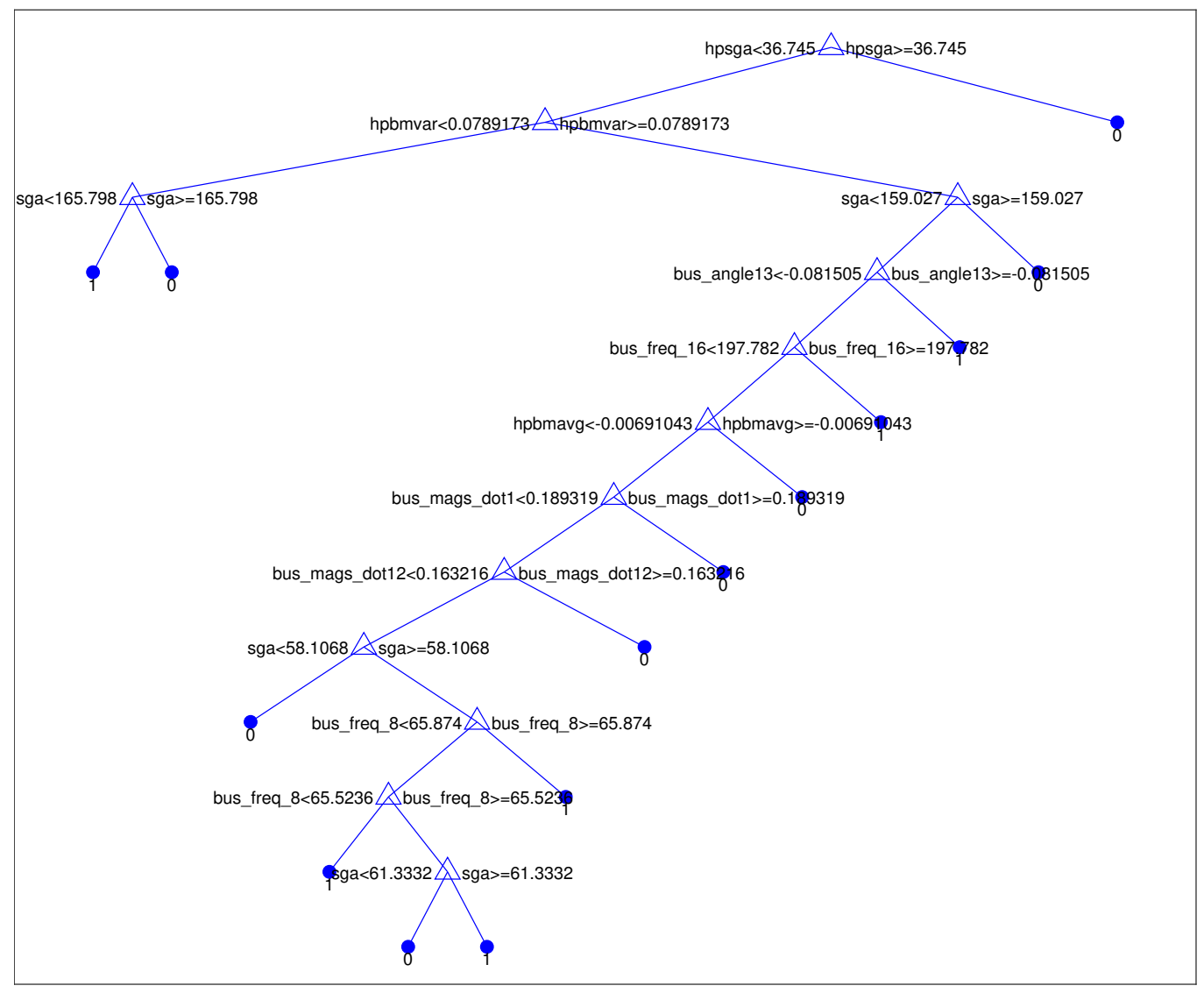

Fig. A.1.: DT representation of control combination 1 


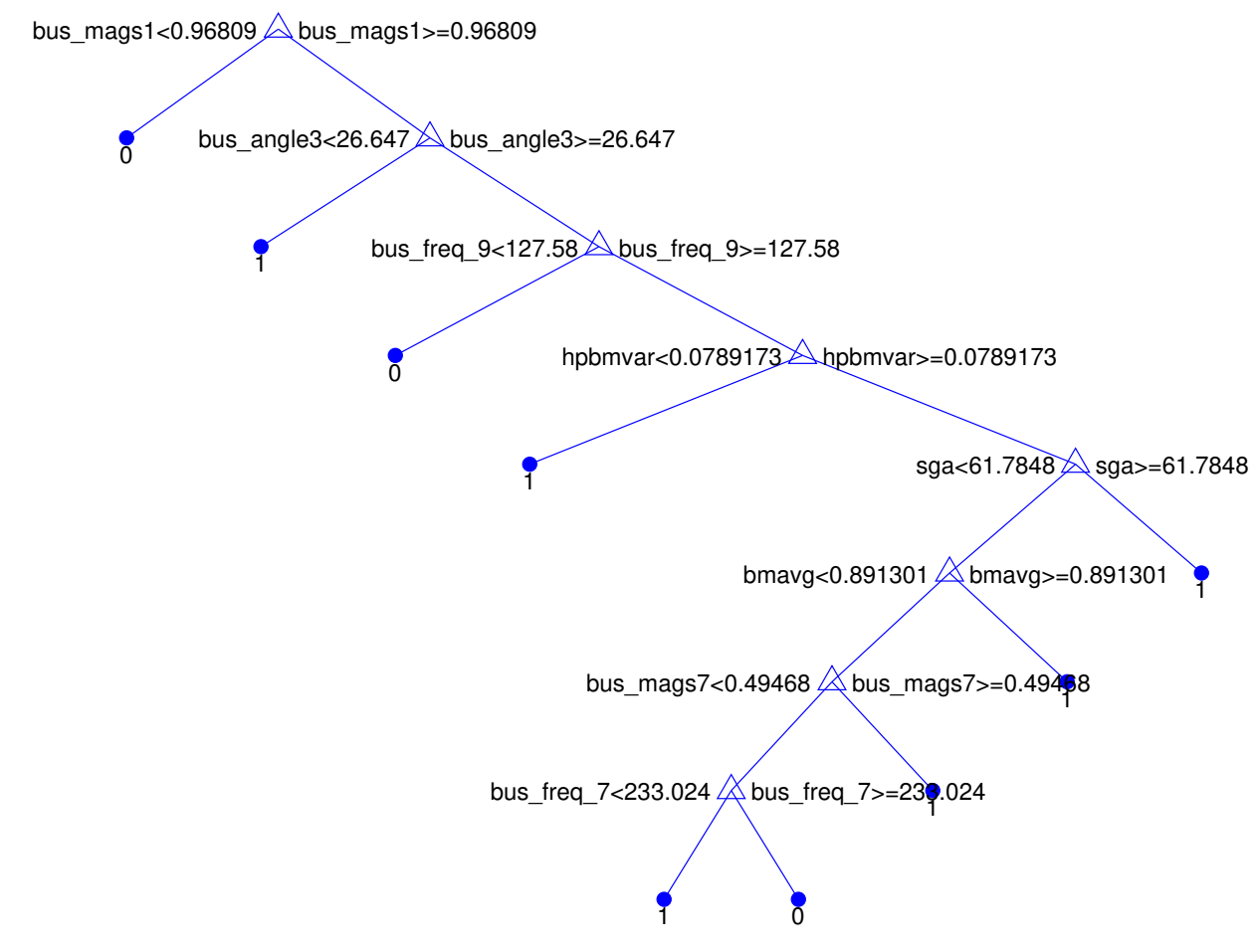

Fig. A.2.: DT representation of control combination 2 


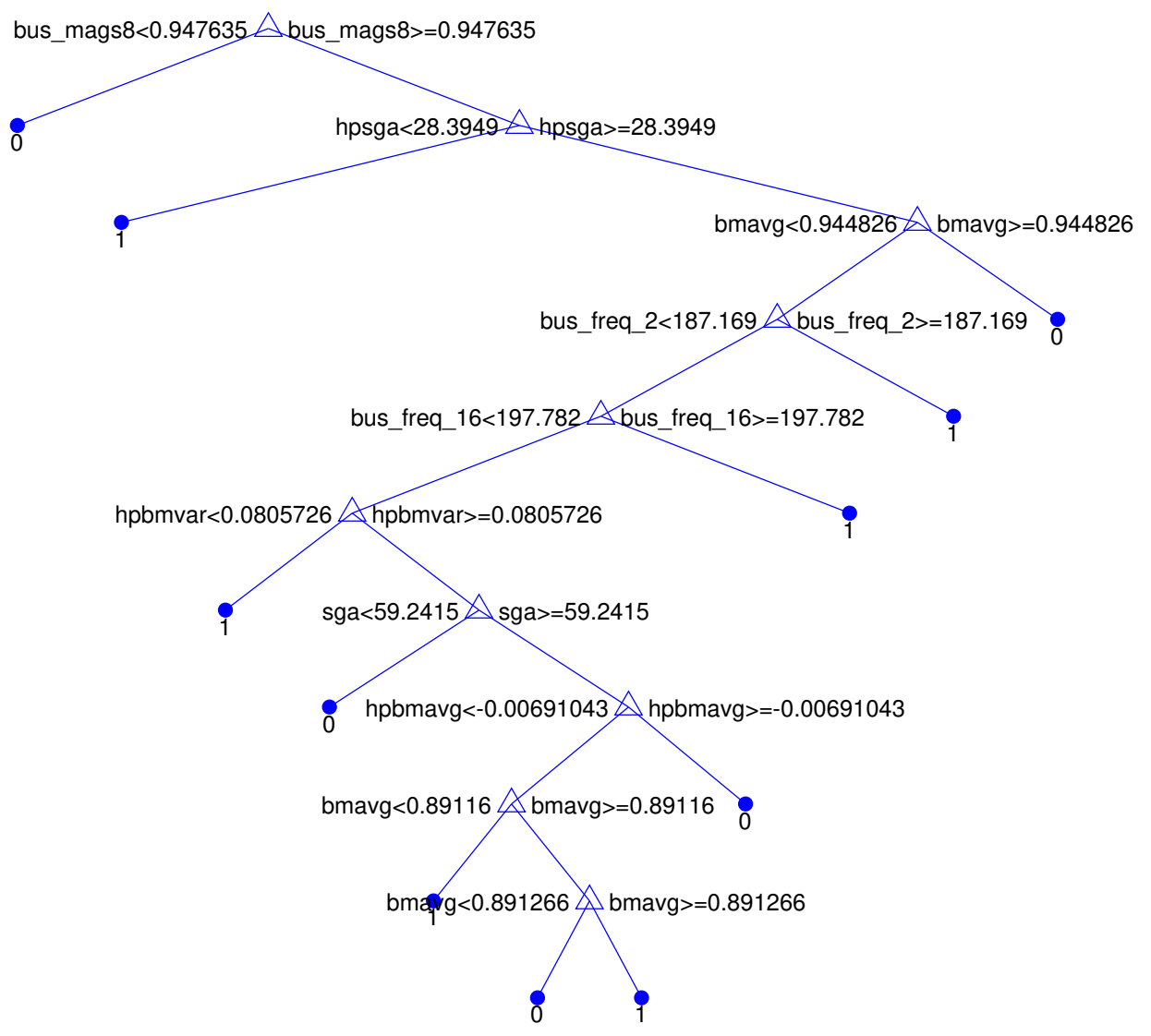

Fig. A.3.: DT representation of control combination 3 